\title{
How plasticity, genetic assimilation and cryptic genetic variation may contribute to adaptive radiations
}

\author{
RALF F. SCHNEIDER and AXEL MEYER \\ Lehrstuhl für Zoologie und Evolutionsbiologie, Department of Biology, University of Konstanz, Universitaetstrasse 10, 78457 \\ Konstanz, Germany
}

\begin{abstract}
There is increasing evidence that phenotypic plasticity can promote population divergence by facilitating phenotypic diversification and, eventually, genetic divergence. When a 'plastic' population colonizes a new habitat, it has the possibility to occupy multiple niches by expressing several distinct phenotypes. These initially reflect the population's plastic range but may later become genetically fixed by selection via the process of 'genetic assimilation' (GA). Through this process multiple specialized sister lineages can arise that share a common plastic ancestor - the 'flexible stem'. Here, we review possible molecular mechanisms through which natural selection could fix an initially plastic trait during GA. These mechanisms could also explain how GA may contribute to cryptic genetic variation that can subsequently be coopted into other phenotypes or traits, but also lead to nonadaptive responses. We outline the predicted patterns of genetic and transcriptional divergence accompanying flexible stem radiations. The analysis of such patterns of (retained) adaptive and nonadaptive plastic responses within and across radiating lineages can inform on the state of ongoing GA. We conclude that, depending on the stability of the environment, the molecular architecture underlying plastic traits can facilitate diversification, followed by fixation and consolidation of an adaptive phenotype and degeneration of nonadaptive ones. Additionally, the process of GA may increase the cryptic genetic variation of populations, which on one hand may serve as substrate for evolution, but on another may be responsible for nonadaptive responses that consolidate local allopatry and thus reproductive isolation.
\end{abstract}

Keywords: adaptive radiation, cichlids, flexible stem, genetic accommodation, genetic assimilation, phenotypic plasticity

\section{Introduction}

The ability of single genotypes to produce varying environmentally-tuned phenotypes during the individuals' development is called phenotypic plasticity (Box 1 - Glossary) (Bradshaw 1965). Its influence on evolution and speciation has been debated controversially for decades (for reviews see: West-Eberhard 1989; Schlichting \& Pigliucci 1998; Price et al. 2003; West-Eberhard 2003; DeWitt \& Scheiner 2004; Pfennig \& Pfennig 2012; Gilbert \& Epel 2015; Oke et al. 2015; Sultan 2015). During the

Correspondence: Axel Meyer, Fax: +49 753188 3018;

E-mail: axel.meyer@uni-konstanz.de twentieth century, insights into how genes and allele frequencies underlie evolutionary change led to a strong focus of evolutionary research on the direct genetic effects on the fitness of individuals (Fisher 1930; Waddington 1942; Williams 1966; Smith 1989; Pigliucci \& Murren 2003; Sultan 2015). However, phenotypes are not only genetically determined $(G)$ but can also be influenced by the environment (E) and their interaction $(\mathrm{G} \times \mathrm{E})$. That is, organisms may respond phenotypically to their environment (Ghalambor et al. 2007; Gilbert \& Epel 2015; Sultan 2015) and even shape their environments themselves (Odling-Smee et al. 1996; Sultan 2015). The classical view is that by 'uncoupling' the phenotype from the genotype to varying degrees, which are subject 


\section{Box 1. Glossary}

\section{Adaptive radiation}

A process through which a multitude of new species evolves rapidly from a single common ancestor by phenotypic segregation into different ecological niches. Adaptive radiations can be induced when a founder population colonizes a new habitat with open niches.

\section{Developmental robustness}

A system is developmentally robust if environmental perturbations or DNA sequence mutations have little effect on developing phenotypes.

\section{Developmental trajectory (in this manuscript)}

Simplified concept of the specific gene regulatory pathway that leads to a trait's (usually adaptive) phenotype. Nonplastic traits only have one developmental trajectory while plastic traits have several in response to an environmental cue. As long as developmental trajectories are regularly expressed in a population, they are expected to remain under stabilizing selection.

\section{Diversification potential}

A property of a population that reflects its probability and degree of diversifying evolution when experiencing ecological opportunity. Simplified, it is the product of the ability of a population to utilize available niches, population attributes that increase its phenotypic evolvability and the probability of evolving reproductive barriers to other populations.

\section{Ecological opportunity}

A population-specific property of an environment composed of niche availability (allowing the population to persist) and niche discordance (causing diversification by differing selective regimes across niches).

\section{Evolvability}

The rate at which DNA sequence mutations can induce phenotypic alterations that can be targeted by selection.

\section{Flexible stem hypothesis}

A developmentally flexible lineage may diverge into derived linages under specific conditions. Variation in derived lineages reflects the developmental flexibility of the ancestor (i.e. its flexible stem).

\section{Genetic accommodation}

A selection-driven process during which a trait becomes more or less plastic and thus less or more heritable, respectively.

\section{Genetic assimilation}

A selection-driven process during which a trait becomes less plastic through its genetic fixation, thus removing its environmental responsiveness.

\section{Phenotypic plasticity}

The capacity of a single genotype to produce varying phenotypes according to external (e.g. environmental) cues (also 'developmental plasticity', when induced phenotypes are irreversible and set during development). Adaptive and nonadaptive plasticity produce phenotypes that are closer to or further away from the fitness optimum, respectively.

\section{Reaction Norm}

A reaction norm depicts a trait's phenotypic expressions of a single genotype across a range of environments. Plotting multiple reaction norms together allows the comparison of the level of plasticity among genotypes. For more details, see, for example, Woltereck (1913) and Schlichting \& Pigliucci (1998). 
to selection themselves, plasticity shields the genotype from the direct effects of natural selection (Schlichting 2004). However, theoretical research and simulation models increasingly and convincingly predict that under certain conditions, phenotypic plasticity might nonetheless facilitate evolution, speciation and even adaptive radiations (Box 2) (e.g. West-Eberhard 2005; Ghalambor et al. 2007; Pfennig et al. 2010; Ehrenreich \& Pfennig 2015; Gilbert \& Epel 2015). Gilbert et al. (2015) suggested that three main hurdles must be overcome to fully incorporate plasticity into evolutionary biology: understanding how commonly plasticity contributes to evolutionary transitions, learning about the molecular mechanisms underlying plasticity, and constructing a theoretical framework to predict population-specific evolutionary consequences of plasticity. Here, we aim to help overcome these hurdles by complementing and elaborating recently published work on genetic assimilation (e.g. Ehrenreich \& Pfennig 2015; Levis \& Pfennig 2016) by expanding upon important concepts about the nature of phenotypic plasticity and then discuss how plasticity may contribute to diversification, speciation and adaptive radiations. In addition, we focus on proposing how molecular mechanisms potentially mediate genetic assimilation and aim to predict patterns of genetic, transcriptional and phenotypic diversity expected in extant derived lineages that have a common phenotypically plastic ancestor. Evidence is summarized concluding that gene regulatory networks underlying plastic traits might provide the developmental variability that can serve as the substrate for the evolution of phenotypic diversity during lineage divergence and establish macro-evolutionary patterns.

\section{The nature of phenotypic plasticity}

Phenotypic plasticity is widespread in nature and has been demonstrated for most evolutionary lineages in ecologically relevant contexts. A multitude of possible environmental cues are known to induce plastic phenotypes (e.g. as summarized in West-Eberhard 2003; Schlichting 2004; Gilbert \& Epel 2015). As plasticity allows organisms to deal with variable environmental conditions, it is assumed to be particularly important for sessile organisms, such as plants (Schlichting 1986; Nicotra et al. 2010; Turcotte \& Levine 2016). However, also in the animal kingdom phenotypic plasticity has been demonstrated in many different contexts such as predator avoidance, seasonal change, social interactions or food availability. Classical examples include Daphnia species that show increased or decreased average body and helmet size when confronted with predator soluble cues or raised at different temperatures, respectively (Ostwald 1904; Dodson 1988). In Hymenopteran insects, phenotypic plasticity was shown to be responsible for the development of the caste phenotypes found in many derived lineages, such as ants or many bees and wasps (Hölldobler 1990). Other well-known examples include swarming behaviour in locusts (Simpson et al. 1999), the induction of cannibalism in spadefoot toads (Pfennig et al. 1993) and sex change in labrid fishes (Warner \& Swearer 1991).

Generally, inducible phenotypes may either vary continuously and depend on the quantity of an environmental stimulus, or they may be discrete and follow an on/off switch mechanism ('polyphenism') (Schlichting \& Pigliucci 1998; Nijhout 2003). Plasticity can thus provide a large pool of phenotypic diversity, even within a population. In spite of plasticity's near ubiquity, the mechanisms by which it may contribute to specialization, lineage diversification and speciation remain debated (but see e.g. Foster et al. 2015; Ghalambor et al. 2015) and some even feel that a new evolutionary paradigm is necessary (e.g. Gilbert \& Epel 2009; Pigliucci \& Müller 2010). It is argued that the level of phenotypic plasticity, as typically depicted in reaction norms, can be the target of selection and thus evolve (Schlichting 1986; Scheiner 1993; Nussey et al. 2005). This implies the existence of mechanisms that increase or reduce a trait's environmental sensitivity and thus reduce or increase its heritability, respectively. West-Eberhard (2003) coined the term genetic accommodation to cover processes in which the level of phenotypic plasticity is either reduced or increased, making the trait's phenotype more or less heritable, respectively (Schlichting \& Wund 2014).

Phenotypic plasticity is common in nature because it often offers advantages to organisms that live in heterogeneous environments and have to deal with varying environmental conditions in which the associated cost of phenotype (i.e. the cost of having a phenotype with suboptimal fitness) is particularly high (West-Eberhard 2003; Murren et al. 2015). Models and empirical studies confirmed that phenotypic plasticity increases geographic distribution ranges and enhances species persistence and fitness, particularly in temporarily heterogeneous environments, as long as the changing environmental cues predict the environmental conditions reliably (Price et al. 2003; Zhang 2005; Chevin et al. 2010; Scheiner \& Holt 2012; Le Vinh et al. 2016; Orizaola \& Laurila 2016). This kind of environmentally induced flexibility may occur in any kind of trait, such as morphology (Greenwood 1964; Pfennig et al. 2002; Binning et al. 2010), life history (Spight \& Emlen 1976; Nylin \& Gotthard 1998; Visser et al. 2009; Foster et al. 2015), and even behaviour (Cotman \& Berchtold 2002; Torres-Dowdall et al. 2012), such as the avoidance of this new environmental cue (e.g. predator smell). Both theoretical as 


\section{Box 2. Cichlids as a model system for adaptive radiations \& convergence}

As one of the most species-rich families of vertebrates and a distribution across three continents, cichlid fishes represent an excellent model to study adaptive radiation (Kocher 2004; Seehausen 2006; Muschick et al. 2012). In Lake Victoria alone, about 500 species have arisen within the last 200,000 years (Meyer et al. 1990). The East African cichlids are particularly famous, as numerous ecological niches have been filled independently but in parallel across the lakes Victoria, Malawi and Tanganyika (Fig. 5 Box 2) (Meyer et al. 1990; Kocher et al. 1993; Meyer 1993a; Stiassny \& Meyer 1999; Schön \& Martens 2004; Young et al. 2009). Besides the adaptive radiations in these three lakes, there are numerous other examples of rapid evolution and adaptive radiations in African cichlids, such as that of the Haplochromines in Lake Kyoga and Tilapiines in Lake Barombi Mbo (Trewavas et al. 1972; Turner et al. 2001). Furthermore, it was demonstrated that the Geophaginae subfamily of the Neotropical cichlids exhibits all signs of an adaptive radiation, as many species emerged within a very short evolutionary time span and the radiating lineages are phenotypically specialists, occupying narrow ecological niches (Lopez-Fernández et al. 2005). In particular, the genus Crenicichla was proposed to present an independent adaptive radiation within the Geophaginae, as they show astonishingly high levels of phenotypic diversification and niche specialization (Piálek et al. 2012). Another example is the Central American Amphilophus spp. complex. These fish inhabit the two geologically old great Nicaraguan lakes, Lake Nicaragua and Lake Managua, but also colonized an assemblage of very young crater lakes (Elmer et al. 2010). Within only a few thousand years, considerable phenotypic divergence has occurred between source populations (inhabiting the two great lakes) and the different satellite populations. Also, speciation has occurred within these satellite lineages, presumably in sympatry (Barluenga et al. 2006; Barluenga \& Meyer 2010).

\section{Convergence in cichlid fishes}

By investigating morphological phenotypes of ecologically relevant traits across the phylogeny of cichlids, it has been repeatedly noted that adaptive phenotypes recur across cichlid radiations, most famously in the three East African Great Lakes (e.g. Kocher et al. 1993; Stiassny \& Meyer 1999; Rüber \& Adams 2001; Muschick et al. 2012). Fish species from different lakes that occupy similar niches often have extremely similar morphological phenotypes. This is particularly noteworthy because these phenotypically similar species do not share a direct common ancestor in whom this morphology was already present, but originate from a single ancestral lineage per lake that also gave rise to all/many other species of the radiation and thus a plethora of phenotypes (Kocher et al. 1993; Salzburger et al. 2005). The coinciding assemblages of phenotypes in several radiations (and thus lakes) were thought to be the result of similar selection regimes in the respective similar ecological niches, leading to the independent evolution of very similar phenotypes, but with independent genetic bases - a textbook example for 'convergent evolution' (Stiassny \& Meyer 1999). Cichlid convergent traits include thick lips, mouth brooding, body coloration and colour patterns, dentition and shape of the oral and pharyngeal jaws, gut lengths, body shape and many more (Muschick et al. 2012; Colombo et al. 2013; Manousaki et al. 2013). The molecular underpinnings of the phenotypic convergence in these traits are mostly yet to be explored.

Fig. 5 Box 2 Simplified cladogram of the 'modern Haplochromine' cichlid fishes. Species diversity mostly comprises the Lake Victoria (green), Lake Malawi (blue) and Lake Tanganyika Tropheini radiations (red) with age estimations approximately around $\sim 0.1$ Myrs, $\sim 1.5$ Myrs and $\sim 2.5$ Myrs, respectively (Salzburger et al. 2005; Elmer et al. 2009; Brawand et al. 2014). All three radiations experienced explosive phenotypic diversification after colonization. Additionally, the mostly riverine generalists Astatotilapia burtoni and Astatoreochromis alluaudi are included. Cladogram following Brawand et al. (2014).
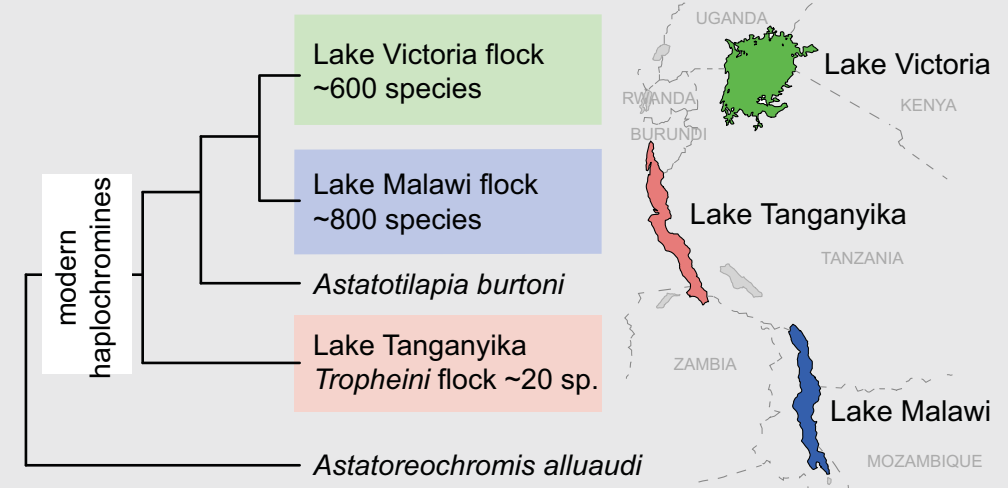
well as empirical studies suggested that phenotypically plastic species are also particularly successful in colonizing new environments, as plasticity can facilitate the immediate exploitation of many different niches (Yeh \& Price 2004; Richards et al. 2006; Thibert-Plante \& Hendry 2011).

In addition to increased species persistence and colonization success, phenotypic plasticity may also indirectly affect the standing genetic variation of populations (Nussey et al. 2005; Pfennig et al. 2010; Renn \& Schumer 2013). As selection acts on phenotypes, genotypes that plastically produce the same adaptive phenotype may shield their genetic differences from selection, which can preserve both genotypic variation as well as newly arising alleles in populations (which contributes to the populations cryptic genetic variability) (Pfennig et al. 2010; Draghi \& Whitlock 2012; Schlichting \& Wund 2014). Plasticity's major advantages hold true as long as the future environmental conditions can be predicted sufficiently reliably from perceived cues. Limits and costs of plasticity may thus arise due to unreliable environmental cues (e.g. Zimova et al. 2016) as well as other factors such as an inherent cost of maintaining the often complex regulatory network necessary for plastic responses, although studies increasingly suggest that the costs of plasticity are probably negligible in most cases (DeWitt et al. 1998; Ancel \& Fontana 2000; Price et al. 2003; Binning et al. 2010; Snell-Rood et al. 2010; Hendry 2015; Murren et al. 2015). Although a certain adaptive phenotype may be (plastically) produced by various genotypes, natural selection will still naturally favour the genotype with lowest associated costs, which in turn may reduce genetic diversity in a population (DeWitt et al. 1998; Pfennig et al. 2010).

As plastic responses can be induced by a variety of environmental stimuli, it is important to distinguish between 'adaptive' and 'nonadaptive' plasticity (Ghalambor et al. 2007). Adaptive plasticity can be interpreted as a phenotypic response to an environmental stimulus, for which certain developmental programmes have been evolved, leading to a phenotype with an increased fitness (i.e. the produced phenotype is closer to the local fitness optimum compared to the generic phenotype) (Schlichting 1986; Price et al. 2003). In contrast, when nonanticipated environmental stimuli induce a plastic response, the resulting phenotypes may be nonadaptive and therefore confer no increased fitness or even reduce fitness relative to the generic phenotype of the organism (Newman 1992; Ghalambor et al. 2007). As natural selection disfavours nonadaptive phenotypes, genotypes with nonadaptive plastic responses are expected to disappear from a population rapidly if the inducing environmental stimulus is common. Nonetheless, genotypes with nonadaptive phenotypic plasticity can presumably be retained in a population as long as their nonadaptive plastic response is not induced. It is thus important to distinguish between adaptive and nonadaptive plastic responses, as they can have different ecological and evolutionary consequences (Ghalambor et al. 2007, 2015). Throughout this manuscript, we refer to the adaptive type of plasticity, if not mentioned otherwise.

\section{Phenotypic plasticity's effect on evolution and speciation}

As plasticity provides increased phenotypic variation, it is likely to also be a prime driver of evolutionary processes such as phenotypic diversification. However, in the era of the 'modern synthesis', phenotypic plasticity was typically seen as being confounding for experiments, as the plastic response altered the phenotypes in a way that is not directly determined by the genotypes (West-Eberhard 1989; Nijhout 2015; Sultan 2015). In the light of this property, the notion arose that plasticity generally reduces the pace of evolutionary change as it partially uncouples the genotype from the phenotype and thus shields the genotype from the force of natural selection. Hence, it would move a trait effectively to an 'evolutionary slower lane' (Stebbins 1950; Williams 1966; Meyer 1987; Bell \& Aubin-Horth 2010; Nijhout 2015).

Contrasting this view, plasticity in key ecological traits has often been found in taxa that are well known for their high rates of evolution and phenotypic diversification (Pfennig \& McGee 2010). Phenotypic plasticity has been described in ecologically relevant traits such as leg length in Caribbean Anolis lizards (Losos et al. 2000), horn length in horned beetles (Emlen 1994; Moczek \& Kijimoto 2014), castes in Hymenopterans (Cahan et al. 2004), body colour in spiny-legged spiders (Brewer et al. 2015), feeding ecology and life history decisions in nematodes (Gutteling et al. 2007; Serobyan et al. 2013), body shape and jaw size in Oceanic sticklebacks (Wund et al. 2008; Foster et al. 2015; Mazzarella et al. 2015) and pharyngeal jaw shape and dentition in cichlids (Box 3: Plasticity in cichlids) (e.g. Greenwood 1964; Meyer 1993b; Muschick et al. 2012). These findings support the notion that phenotypic plasticity in ecologically relevant traits rather promotes evolution and phenotypic diversification, which eventually may even facilitate adaptive radiations (West-Eberhard 2003; Wund et al. 2008; Pfennig et al. 2010). Famously, early in the history of evolutionary biology, it was hypothesized that originally plastic traits can become genetically fixed in a homogeneous environment, where the advantage of being plastic is lost (Waddington 1942, 1953; West-Eberhard 2003). 
As whole sets of originally plastically induced phenotypes may be fixed, explosive phenotypic diversification may be explicable in this way (West-Eberhard 2003).

The first empirical support for a plastic trait becoming heritable was shown in the 1950s by Waddington (1953). He demonstrated that his results were most likely not due to selection on the genotype and proposed a new mechanism that he called 'genetic assimilation'. During the process of genetic assimilation, an originally environmentally induced phenotype becomes secondarily fixed and thus consistently expressed - that is, a trait loses its plasticity (Waddington 1942, 1953, 1961). It was proposed that due to the inherent cost of plasticity, variants with a reduced level of phenotypic plasticity that still includes the selected phenotype may have a fitness advantage and would therefore be selectively maintained in a homogeneous environment (DeWitt et al. 1998; Ancel \& Fontana 2000; DeWitt \& Scheiner 2004). Since then, however, only few studies have convincingly shown that genetic assimilation occurred (e.g. Sikkink et al. 2014) or that plasticity is generally connected to inherent maintenance costs. We are far from a complete understanding of the molecular mechanisms that might account for genetic assimilation (Ehrenreich \& Pfennig 2015).

\section{Ancestral phenotypic plasticity may promote explosive speciation events}

It has been proposed for many adaptive radiations that the ancestral lineage might have been particularly phenotypically plastic in key evolutionary traits, that is, adaptive traits that are especially diverse in derived lineages and probably contributed to diversification (Losos et al. 2000; Nylin \& Wahlberg 2008; Wund et al. 2008; Tebbich et al. 2010; Muschick et al. 2012; Brewer et al. 2015; Susoy et al. 2015). This so-called flexible stem hypothesis is a key concept proposing that ancestral phenotypic plasticity can promote speciation and may even have contributed to adaptive radiations (WestEberhard 2003). In the following paragraphs, we will describe why plastic ancestors are likely to facilitate the evolution of adaptive radiations and provide a model of how speciation patterns might be expected to proceed for adaptive radiations derived from an ancestral flexible stem lineage (Fig. 1).

\section{Ecological opportunity and ecotype formation}

A novel environment colonized by a population offers ecological opportunity to this population, a driver of the adaptive diversification of lineages (Schluter 2000; Wellborn \& Langerhans 2015). The magnitude of ecological opportunity is thought to be reflected by the number of available and utilizable niches (niche availability) and the degree to which they differ from each other in their selective regimes to facilitate diversifying selection (niche discordance) (Wellborn \& Langerhans 2015). When comparing the ecological opportunity of a plastic and a nonplastic population that are about to colonize a new environment, the plastic population is predicted to be ready to utilize a broader range of niches if these overlap with their phenotypic plastic range (i.e. width of reaction norm). Both niche availability and niche diversity (and hence the chance for niche discordance) are thus expected to be higher for a plastic population compared to its nonplastic counterpart. Nonplastic lineages can only persist in the new habitat in niches similar or equal to its previous one (Pfennig et al. 2010), and the potential for adaptive diversification is therefore expected to be relatively low compared to a plastic population.

\section{Lineage divergence and adaptive radiation - a flexible stem}

For speciation and adaptive radiations to occur, the colonizer has to undergo (potentially multiple times) lineage divergence. After colonizing a new habitat, a plastic population may form subpopulations, each occupying one available niche and expressing the niche-specific most suitable (i.e. adaptive) plastic phenotype. However, whether a lineage is likely to undergo an adaptive radiation does not only depend on ecological opportunity, but also on the diversification potential of the founding population, which describes the ability of a population to respond to ecological opportunity (Wellborn \& Langerhans 2015). According to Wellborn \& Langerhans (2015), the diversification potential of a population is the product of population properties that facilitate species persistence, properties that increase phenotypic variance when encountered ecological opportunity and properties facilitating reproductive isolation among subpopulations. As phenotypic plasticity promotes species persistence and leads to rapid phenotypic differentiation in different niches (if they correspond to the plastic range), two of three properties confer high diversification potential on a plastic population. The third property is the potential to establish reproductive isolation. Here, it is the plasticity itself that drives spatial segregation of subpopulations and therefore promotes reproductive isolation. Subsequently, further processes such as assortative mating (Doebeli \& Dieckmann 2003), differences in reproductive timing or very high territoriality, may strengthen reproductive barriers (Dieckmann \& Doebeli 1999). A plastic population is thus likely to have a high potential for diversification and therefore is more likely to initiate an adaptive radiation in (macroscopic) sympatry. 


\section{Box 3. Plasticity in cichlids is widespread}

Within the family of cichlid fishes, Greenwood was among the first to scientifically describe phenotypic plasticity in an adaptive trait (Greenwood 1964). He noticed that the morphology of the pharyngeal jaw bones in the generalist species Astatoreochromis alluaudi, which lives in Lake Victoria and the surrounding river system, varies in shape, robustness and dentition depending on population. The main difference between the populations turned out to be the stomach content composition of the fish, having populations with the highest proportion of food being insect larvae (soft food items) and some with the highest proportion being snails (a hard food item, as shells have to be crushed during ingestion). He conducted feeding experiments and found that the robustness of the food items individuals ingest determines the phenotypes of their pharyngeal jaws, which was confirmed in later studies (Greenwood 1964; Hoogerhoud 1986; Gunter et al. 2013).

Since then, phenotypic plasticity in cichlids has been reported in a number of traits. Importantly, plastic species were often reported to be riverine or being part of very recent intralacustrine adaptive radiations (Greenwood 1964; Hoogerhoud 1984; Meyer 1989; Chapman et al. 2001; Hofmann 2003; Swanson et al. 2003).

\section{Patterns of phenotypic plasticity in the pharyngeal jaws of modern Haplochromines}

Astatoreochromis alluaudi belongs to a nonradiated lineage that is directly basal to the 'modern Haplochromines' (Salzburger et al. 2005) (Fig. 5 Box 2). These comprise three adaptive radiations, two of them outstanding as they led to several hundred species within incredible short evolutionary time spans (Meyer 1993a; Salzburger et al. 2005). As mentioned before, the hyperdiverse phenotypes found across species in each of these radiations are famous and convergence was described in a suite of traits (O'Quin et al. 2010; Muschick et al. 2012; Colombo et al. 2013). Previous studies showed that besides in A. alluaudi, pharyngeal jaw phenotypic plasticity can also be found in Haplochromis iris and Haplochromis ishmaeli, young, radiating species from the Lake Victoria flock that specialized on insects and snail cracking, respectively, and Astatotilapia burtoni (Smits et al. 1996). The latter is a generalist that inhabits Lake Tanganyika and associated river systems and is phylogenetically situated either between the Tropheini radiation and the Lake Malawi radiation (Brawand et al. 2014), or between the latter and the Lake Victoria radiation (Fig. Box 2) (Salzburger et al. 2005). Data recently collected in our laboratory suggest that the levels of inducible adaptive and nonadaptive plasticity vary considerably between representatives from the radiations and the rivers (H. M. Gunter, R. F. Schneider, I. Karner, C. Sturmbauer \& A. Meyer, manuscript in prep.).

Indeed, a considerable body of literature supports the notion that many adaptive radiations may have begun in sympatry (e.g. Kawecki 1997; Dieckmann \& Doebeli 1999; Kondrashov \& Kondrashov 1999; Doebeli \& Dieckmann 2003; Levis \& Pfennig 2016). Through the process of genetic assimilation, emerging lineages may then come to fix the most adaptive phenotype for their niche. Arising phenotypes of radiating lineages are thus expected to initially reflect different phenotypes of the ancestral range of plasticity (Levis \& Pfennig 2016). Depending on the number of emerging lineages and the overall morphospace they occupy, a large number of new species can potentially arise within a short evolutionary time span. Indeed, the reduction of plasticity in a lineage has often been correlated with speciation events (Schwander \& Leimar 2011), potentially reflecting plasticity-mediated diversification events and subsequent genetic assimilation of phenotypes. In contrast, many nonadaptive radiations arose presumably due to allopatric divergence of the ancestral lineage without a major initial niche shift in descending lineages (Nei et al. 1983; Gittenberger 1991; Rundell \& Price 2009). Thus, the contribution of plasticity to diversification is expected to be reduced in nonadaptive compared to adaptive radiations, as a sympatric adaptive radiation is thought to depend on the availability of numerous exploitable niches in the same habitat. In addition, a plastic lineage may have increased standing genetic diversity (as discussed above), which can serve as the substrate for subsequent adaptations (Barrett \& Schluter 2008).

\section{Repeated colonization of similar habitats - convergence and parallel evolution}

When a phenotypically plastic stem lineage (the 'source' population) persists over longer geographic time, it may encounter the opportunity to colonize further similar habitats. Such parallel colonizations may lead to repeated fixation of similar phenotypes if colonized habitats offer similar niches for which a specific, plastically induced adaptive phenotype exists. Such 


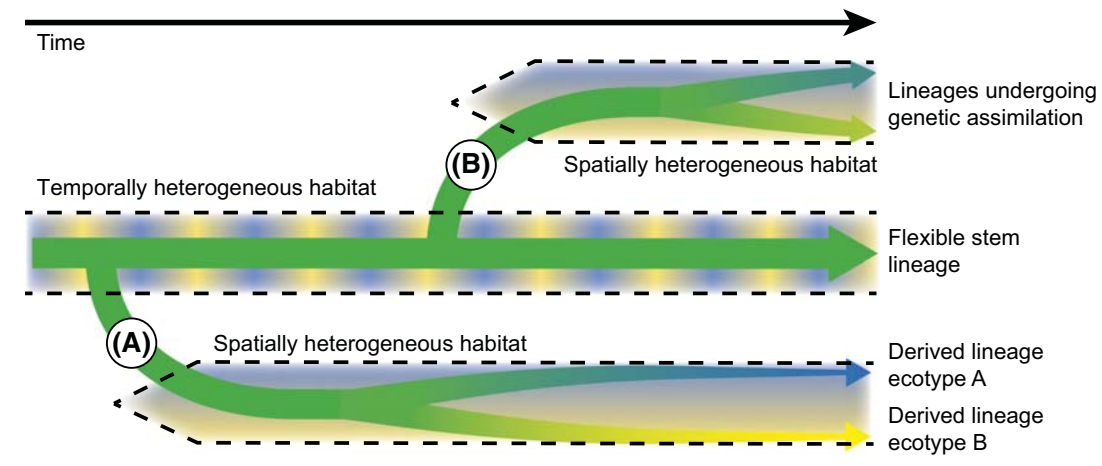

Fig. 1 Illustration of the flexible stem model. A phenotypically plastic lineage (flexible stem) inhabiting a temporally heterogeneous habitat (e.g. heterogeneity in abundance of alternative food types in a river) colonizes a spatially heterogeneous habitat in which the two food sources are spatially separated (e.g. lake habitat with one food-type associated to the shore and the other to the open water) (A). Here, subpopulations displaying the alternative adaptive plastic phenotypes, and population divergence can occur accordingly. Gradually, selection fixes the expressed phenotype in each population via genetic assimilation, effectively reducing the level of plasticity in each emerging lineage. Later in time, the same flexible stem lineage might colonize another similar habitat, offering the same niches (e.g. another lake) (B). Again, lineage divergence can occur according to the two niches, leading to a phenotypically very similar species assemblage as in the other habitat (A) due to their common ancestor's phenotypic range. Convergent/parallel phenotypes between derived lineages of separate colonizations are thus initially expected to be very common.

scenarios were proposed for oceanic stickleback populations that colonized freshwater lakes, whitefish in postglacial lakes and for Darwin's finches on the Galapagos Islands (Robinson \& Parsons 2002; Wund et al. 2008; Tebbich et al. 2010; Lundsgaard-Hansen et al. 2013; Oke et al. 2015). For example, marine sticklebacks are phenotypically plastic and can express phenotypes along a benthic (deeper body with larger eyes) to limnetic (elongated body with smaller eyes) phenotype gradient. While colonizing freshwater habitats, these sticklebacks were presumably confronted repeatedly with two different freshwater lake niches, a limnetic one in shallow lakes and a benthic one in deeper lakes, each with a specific selective regime (Schluter 1993; Wund et al. 2008). The morphologies of a limnetic and a benthic stickleback population that were collected in two Alaskan lakes were found to qualitatively reflect the plastically inducible limnetic and benthic phenotypes of a marine population that approximates the common marine ancestor of the freshwater populations (Wund et al. 2008). Repeated induction of ancestral plastic phenotypes and their subsequent (partial) fixation may thus provide an explanation for some instances of the phenomenon of recurring phenotypes among independent colonizations of similar habitats (Oke et al. 2015). In the following sections, we will explore possible molecular mechanisms, and their traces, that can potentially mediate the process of genetic assimilation and how it may overcome the uncoupling of genotype and phenotype that characterizes a plastic trait.

\section{Gene regulatory networks underlying plasticity may provide a prime substrate for phenotypic diversification}

Environmentally induced plastic phenotypes are thought to be controlled by gene regulatory networks (GRNs) (Schlichting \& Pigliucci 1993) that often remain poorly understood, particularly in nonmodel organisms (Pfennig \& Ehrenreich 2014). GRNs appear to often have a modular structure, i.e. sets of genes that are more or less jointly up- or downregulated, for example during the plastic response (Shiga et al. 2007; Wagner et al. 2007; Aubin-Horth \& Renn 2009; Aubin-Horth et al. 2009; Hinman et al. 2009; Schneider et al. 2014; Gilbert \& Epel 2015). At some point during their evolution, GRNs underlying plastic traits have acquired responsiveness to external stimuli that trigger new regulatory cascades leading to new adaptive phenotypes (via alternative developmental trajectories). A particularly wellunderstood example for a gain of plasticity can be found in the nematode Pristionchus pacificus, in which a recent gene duplication of a sulfatase (eud-1) was identified to be the main switch between the developmental trajectories underlying two distinct plastic feeding morphologies (Ragsdale et al. 2013). Expression of the eud-1 gene is regulated via histone methylation and expression of an antisense RNA (Serobyan et al. 2016). These regulatory mechanisms are presumably connected to a hormone and its receptor, and hormone levels are directly influenced by pheromone concentrations in the environment (Bento et al. 2010). 
Developmental switches that respond directly or indirectly to environmental stimuli allow a single genotype to evolve multiple developmental trajectories in parallel, leading to alternative phenotypes. GRNs of plastic traits are thus outstanding in that they not only allow different genotypes to produce the same phenotype (and thus contribute to developmental robustness), but also to comprise multiple developmental trajectories leading to multiple (plastic) phenotypes within a single genotype. Therefore, they provide a twofold opportunity for the evolution of phenotypic diversity during lineage divergence and specialization (e.g. during an adaptive radiation): (i) an ancestral GRN of a plastic trait can allow diverging lineages to immediately exhibit unique adaptive phenotypes fitting their respective niches by utilizing different developmental trajectories. Immediate phenotypic diversity among emerging lineages would thus reflect (partially) the plastic range of the ancestor (as predicted by the flexible stem hypothesis). (ii) Utilizing only one developmental trajectory (or a subset) in a specialized derived lineage renders all other trajectories of that genotype unused. However, with time, these 'dispensable' trajectories may provide an additional substrate for longer term phenotypic change (as they are a source of cryptic genetic variation).

The following sections illustrate (i.) how selection can potentially genetically fix a plastic phenotype via genetic assimilation in a constant environment. (ii.) We then summarize predicted patterns on genetic, transcriptional and phenotypic variability that are expected to arise from diversification based on a plastic trait. (iii.) Finally, we speculate on how phenotypes can expand phenotypically from the ancestral phenotypic range in derived lineages, potentially more rapidly than classically assumed.

\section{Step-wise degeneration of noninduced developmental trajectories - use it or lose it}

In constant environments, plastic traits may undergo genetic assimilation (Fig. 2): if an environmentally induced phenotype is constantly expressed (across generations), only the specific developmental trajectory underlying the specific phenotype is in usage and thus can be the target of stabilizing (or directional) selection (Price et al. 2003; Lahti et al. 2009). At this stage, mutations within the expressed developmental trajectory can only be selected for if these mutations lead to phenotypes beyond the ancestral plastic range and if these new phenotypes have higher fitness values. However, if the fitness optimum lies within the ancestral plastic range, plasticity is predicted to shield otherwise beneficial mutations from selection. In this simplified example, with no inherent cost of plasticity, natural selection can therefore not directly contribute to a trait's genetic fixation as long as the optimal phenotype is within the ancestral range. To explain how genetic assimilation can occur under such conditions, the remaining parts of the GRN have to be considered. The developmental trajectories underlying noninduced phenotypes are not expressed and thus released from stabilizing selection ('relaxed selection'), a prerequisite for cryptic genetic variation to be maintained (Rutherford \& Lindquist 1998; Lahti et al. 2009). Thus, these unused developmental trajectories of the GRN (coding and regulatory sequence) are predicted to be prone to accumulate random mutations and thus degenerate (Fig. 2D,F, H) (Masel et al. 2007). In addition, regulatory mutations consolidating the expression of the adaptive phenotype expressed in the given constant environment are not selected against, as would be the case in a heterogeneous environment, and can thus persist (Fig. 2C,E,G). Therefore, the more extensive a GRN underlying a plastic trait is, the more cryptic genetic variation can accumulate when the trait undergoes genetic assimilation that is predicted to increase the population's evolvability (Iwasaki et al. 2013). This is in line with theoretical work generally indicating that developmental robustness increases the evolvability of populations (Wagner 2008; Hayden et al. 2011).

Generally, degeneration of regulatory circuitry may affect coding and noncoding sequences differently: as many genes are pleiotropic, coding regions presumably often remain under stabilizing selection due to their contribution to other traits (Jarvela \& Hinman 2015). In contrast, regulatory regions are expected to be often less pleiotropic (Wray 2007), particularly if they contribute only to one trait's phenotype. Thus, if an inducible phenotype is not expressed, its specific regulatory sites are not under stabilizing selection and can more easily degenerate through the accumulation of neutral mutations (in the given homogeneous environment). Even single point mutations in regulatory sites of genes can have switch effects on the genes' sensitivity towards individual transcription factors, particularly in character loss (Van Laere et al. 2003; Mayo et al. 2006; Hoekstra \& Coyne 2007). Alterations in cis- and trans-regulatory sites may have different effects, as cis-sites are typically thought to regulate the expression of one gene, while trans-sites can often regulate many genes (as discussed in Ehrenreich \& Pfennig (2015)). Considering the modular structure of many GRNs, a change in expression level of a single key transcription factor induced by mutations in regulatory sites may have tremendous effects, as whole gene modules can be affected (Fig. 3). Across generations, even few of these mutations therefore have the potential to degenerate the noninduced 


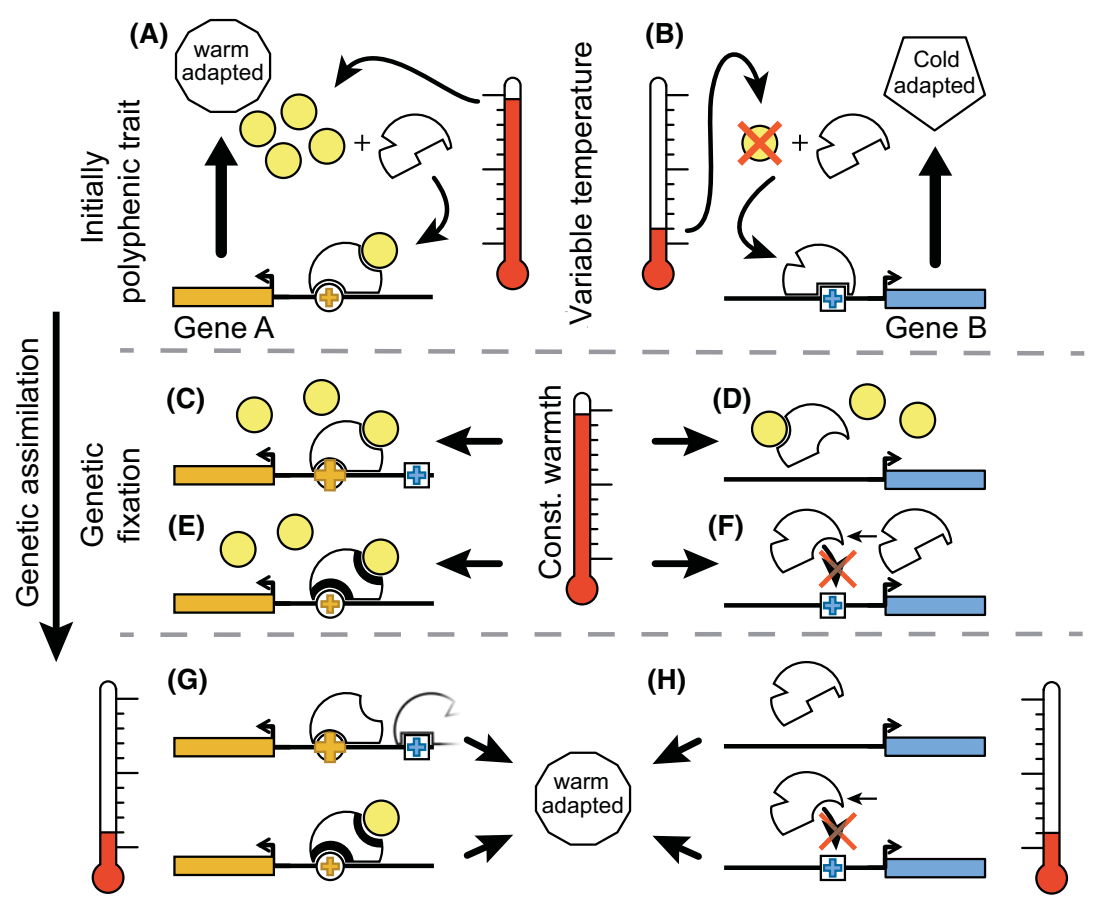

Fig. 2 Simplified examples of how genetic assimilation may genetically fix a previously environmentally responsive trait. A plastic trait's expression is determined by gene products A and B, while A is inducing a phenotype adapted to warmth and B inducing the cold adapted one. A and $\mathrm{B}$ transcription is induced by the same transcription factor ' $\mathrm{TF}^{\prime}$. It binds to its binding site in the promoters of gene A, if a cofactor (yellow circle) is present 'TF-A' (A). If no cofactor is present, 'TF-B' will bind to another binding site in the promoter of gene B (B). Temperature (environmental stimulus) positively regulates the cofactor's abundance. Across time, constant warmth may lead to genetic assimilation via degeneration of the developmental trajectory of the cold adapted phenotype and consolidation of the warm adapted phenotype. Random mutations may, for example (C), increase binding affinity of TF-A to the gene A promoter via TF site modification or allow TF-B binding by the appearance of TF-B binding sites or (D) lead to loss of TF-B binding sites in the gene B promoter. Furthermore, random TF modifications may (E) increase binding affinity of the cofactor to the TF or the TF-A to its binding site or (F) lead to changes in the binding motives of TF-B, for example by allowing it to bind to the gene A promoter even without co-factor. After such events, even in low temperature environments, the warm adapted phenotype is produced due to consolidation of its developmental trajectory $(\mathrm{G})$ and degeneration of the cold adapted phenotype's developmental trajectory. As the trait becomes genetically fixed, natural selection may increasingly act on the gene sequence of gene A.

phenotypes' trajectories or its 'inducibility' by environmental stimuli (Fig. 3B,C). Populations undergoing genetic assimilation for a certain phenotype may therefore be particularly dependent on homogeneous environmental stimuli, as alternative stimuli may induce malfunctional developmental trajectories potentially leading to maladaptive phenotypes. In this way, genetic assimilation may contribute to population reproductive isolation.

Although genetic assimilation may lead to rapid phenotypic divergence, genetic fixation by initially neutral mutations may not be a very fast process, as it relies on random mutations. However, faster genetic assimilation may occur if there are maintenance costs of plasticity: phenotypically plastic traits may require costly and elaborate regulatory networks that respond to variable environmental stimuli by inducing a fitter phenotype (DeWitt et al. 1998). If environmental conditions are rather homogeneous, maintaining such a regulatory network offers no fitness advantage and natural selection is thus expected to select for genotypes with reduced 'maintenance costs', that is reduced plasticity (Pigliucci et al. 2006). Costs can thus promote genetic fixation in homogeneous environments. In addition, trans-generational epigenetic mechanisms may be another mechanism facilitating genetic assimilation: environmentally induced phenotypes in a parental generation may be epigenetically inherited by offspring generations, even in the absence of the inducing environmental cue (Whitelaw \& Whitelaw 2006; Aubin-Horth \& Renn 2009; Mirouze \& Paszkowski 2011; Schlichting \& Wund 2014; Beaty et al. 2016). Thus, it can be speculated that epigenetic inheritance of plastically acquired phenotypes may buffer against short-term environmental heterogeneity that would elsewise interfere with ongoing genetic assimilation (Jablonka \& Lamb 2007). 
(A)

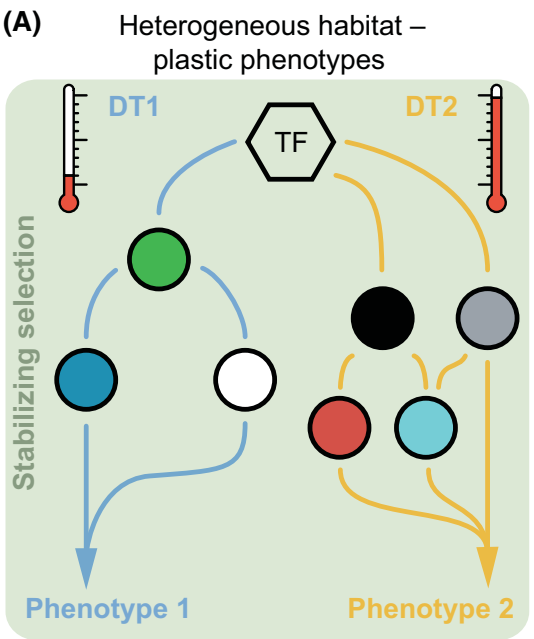

(C) Degenerated plastic response
(B) Permanently homogeneous habitat genetically fixed phenotype 2

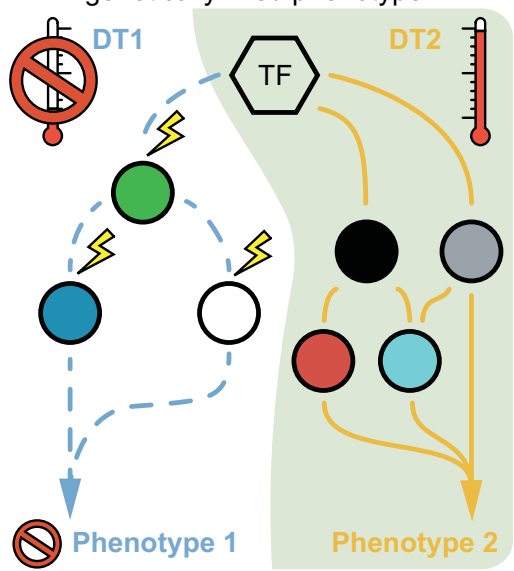

(D) Cooption of moduls from DT1 into DT2

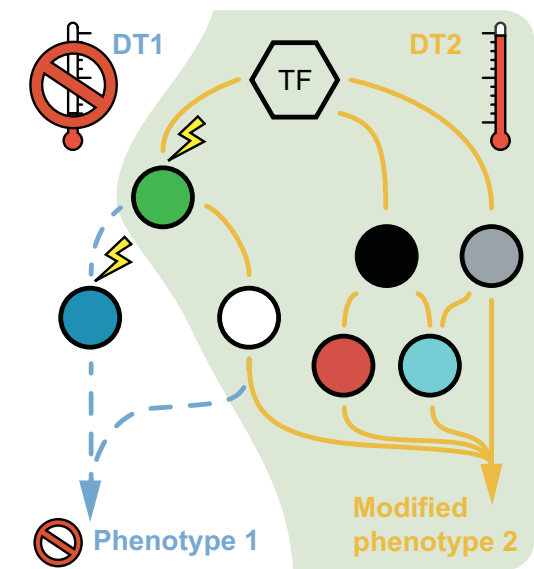

Fig. 3 Illustration of how genetic assimilation may fix and alter a trait by the degeneration and reutilization of developmental trajectories. In a heterogeneous environment, the environmental cue varies (here: temperature) (A). In a phenotypically plastic population, the environmentally controlled transcription factor ' $\mathrm{TF}^{\prime}$ controls a trait's phenotype: low temperatures induce expression of developmental trajectory 1 (DT1, blue lines, left side) and high temperatures induce developmental trajectory 2 (DT2, orange lines, right side) which lead to the adaptive phenotypes 1 and 2, respectively. The environmentally responsive portion of the trait's underlying regulatory network consists of different genes (or gene modules, illustrated as circles) in each DT. As both DTs are regularly expressed and adaptive, both are under stabilizing selection. After the environment changed to permanent warmth, only DT2 was induced and remained under stabilizing selection (B). In contrast, DT1 fell prone to random mutations, which successively degenerated the circuitry of the involved gene regulatory network (GRN). When a population undergoing genetic assimilation by DT degeneration is confronted with the original plasticity cue (here: coldness), the induced phenotype 1 may be already degenerated and less adaptive, as parts of its underlying DT are not functional anymore (C). Parts of a degenerating DT may be reutilized by other DTs of the same or other traits, potentially facilitating the evolution of phenotypes beyond the ancestral range (D).

Ancestral plasticity's traces in genetic, transcriptional and phenotypic patterns during lineage diversification

A promoting effect of ancestral phenotypic plasticity to lineage diversification seems plausible, for example in the context of colonization events, and it may indeed facilitate adaptive radiations (Levis \& Pfennig 2016). Present adaptive radiations (and particularly repeated adaptive radiations) may offer the opportunity to test for the effects of ancestral plasticity, as the traces of (ongoing) genetic assimilation are predictable and should be observable in today's radiations.

The process of genetic assimilation and the accompanying degeneration of alternative phenotypes is a form of regulatory evolution. Generally, regulatory evolution is thought to be a main driver of phenotypic evolution, essentially by the rewiring of existing GRNs ('developmental recombination' sensu West-Eberhard 2005) (e.g. Britten \& Davidson 1971; King \& Wilson 1975; WestEberhard 2005; Davidson \& Erwin 2006; Prud'homme 
et al. 2007; Wray 2007; Carroll 2008). Regulatory evolution may include sequence evolution of cis-regulatory elements in the genome (such as enhancers, promoters, splice-site modules and microRNAs) but also coding sequence evolution that can affect the binding properties of transcription factors as well as changes of epigenetic patterning and processing (e.g. alterations in DNA or histone methylation or acetylation). These mechanisms can work synergistically to shape new developmental trajectories, as exemplified in the feeding polyphenism of Pristionchus pacificus mentioned before (Ragsdale et al. 2013; Serobyan et al. 2016). DNA sequence mutations can alter interactions between regulatory pathways and thus lead to phenotypic change (Alonso \& Wilkins 2005). Thus, rewiring of existing developmental pathways, such as those underlying plastic responses, can potentially contribute to fast lineage divergence in phenotypes induced by only minor underlying genetic divergence (West-Eberhard 2005). Based on gene reaction norms, Renn \& Schumer (2013) provided a theoretical framework that assists in classifying evolved gene expression patterns found after genetic accommodation in plastic (behavioural) traits. Complementing this, we focus in the following sections on patterns of phenotypic and transcriptional variation predicted to arise between and within populations undergoing genetic assimilation.

Investigating the contribution of regulatory evolution to the diversification of target lineages can be challenging, as one has to rely on indirect evidence (Levis \& Pfennig 2016). West-Eberhard (2005) described peculiar patterns in genes and their expression as well as morphology and behaviour that are expected to emerge across diverging lineages if regulatory evolution is involved in driving lineage divergence. Summarized, these are that (i) across diverging lineages, homologous genes are expected to be conserved over relatively distantly related taxa (although they might be used in different contexts), (ii) in emerging lineages, phenotypic divergence may be relatively large while genetic divergence remains subtle, (iii) among closely related species, homoplasy and parallelism are common, and (iv) phenotypic differences in key traits across emerging species can arise before reproductive isolation.

In addition to these four predictions that presumably apply to all cases in which regulatory evolution drives diversification, further predictions can be made if the underlying GRN is that of an initially plastic diversifying trait and if diversification occurs recurrently. Then, observable convergent/parallel phenotypes are expected to reflect the plastic range of the ancestor, as predicted by the flexible stem hypothesis (West-Eberhard 2003). Diverging lineages that emerge from a plastic ancestral population and occupy different niches thus may not only show marked phenotypic divergence before genetic divergence and reproductive isolation, but also pronounced transcriptional divergence as a result of alternative developmental trajectory usage (Figs 4A-C and 1). Under certain conditions, a flexible stem lineage may have the opportunity to independently give rise to multiple lineages that undergo genetic accommodation for essentially the same ecological niche. Although such events may be rare in sympatry, it may be a common occurrence if the lineages live in allopatry. This may happen if a flexible stem lineage colonizes multiple new habitats with similar ecological niches, as was suggested for marine sticklebacks colonizing freshwater lakes and riverine cichlids colonizing lakes (Fig. 1) (West-Eberhard 2003; Wund et al. 2008).

As these lineages undergo genetic assimilation for the essentially the same phenotype, they are likely to utilize the same ancestral developmental trajectory as well. Thus, we expect that the variation in the transcriptional profiles of genes specific (or with specific expression patterns) to the focal trait is low among the lineages with parallel phenotypes compared to variation found across genes not specific to the focal trait's phenotype. Accordingly, expression profiles of genes specific to a focal trait's parallel phenotypes originating in a common flexible stem are predicted to cluster together, when analysed comparatively with genes that do not contribute to that trait. Or, from another perspective: if transcriptional profiles of genes contributing to such a trait's phenotypes are analysed across radiating lineages (with differing phenotypes), a clustering according to phenotypes and not phylogeny is the expected outcome. Thus, we conclude that comparative analyses of gene expression profiles underlying - or not underlying - a focal trait's phenotypes can be a powerful approach to unveil regulatory relationships due to a common flexible stem.

One way to characterize the different developmental trajectories underlying the inducible phenotypes of a plastic trait are their gene expression profiles and their differences (as reviewed e.g. in Gibson 2008). During genetic assimilation, selection successively fixes a specific developmental trajectory by reinforcing its expression or degenerating the developmental trajectories underlying alternative noninduced phenotypes. However, as this process is gradual, we assume that the GRN retains some inducibility by alternative environmental stimuli until the phenotype is entirely fixed. If an individual belonging to a population undergoing genetic assimilation for a certain adaptive phenotype 'A' is confronted with an environmental stimulus (e.g. due to environmental perturbations) that was only experienced by their plastic ancestor and led here to phenotype ' $\mathrm{B}$ ', the derived individual's GRN may still 
(A) Genetic divergence

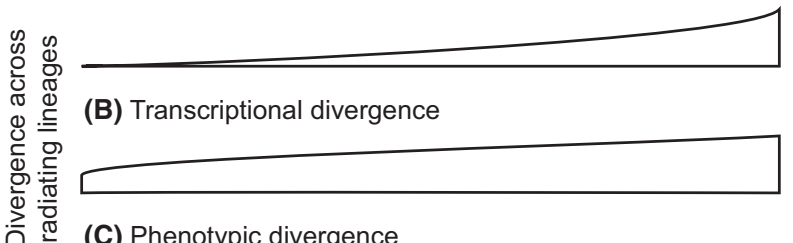

(C) Phenotypic divergence

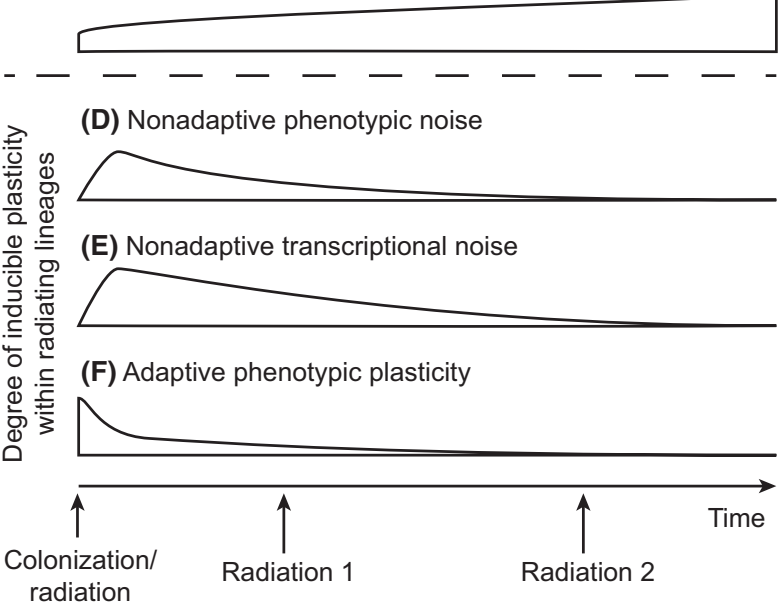

Fig. 4 Patterns of phenotypic and transcriptional variability across diverging lineages expected due to genetic assimilation after a colonization event and during an adaptive radiation with a plastic ancestor. Genetic divergence arises gradually and relatively slowly as it relies on random mutations (A). Different environmental stimuli immediately lead to pronounced differences in transcription across lineages that underlie the respective distinct phenotypes $(B, C)$. Genetic assimilation degenerates unused developmental trajectories gradually $(\mathrm{D}, \mathrm{E})$. Early in this process, some unused trajectories are still responding to the alternative stimuli, however, already low levels of degeneration may lead to nonadaptive transcriptional noise. Accordingly, the resulting phenotypes might only be partially functional and thus result in nonadaptive phenotype characteristics (phenotypic noise). The functionality of the regulatory network underlying the adaptive plastic response is strongly decreasing with the increasing degeneration of alternative developmental trajectories by random mutations in regulatory regions (F). Comparisons of such patterns in repeated radiations of different ages can allow conclusions about the existence of a common flexible stem. Thus, by investigating patterns of inducible plasticity in a trait, it is possible to estimate at which stage in genetic assimilation a radiation is, as indicated in the figure (Radiation 1 vs. Radiation 2).

partially respond to that stimulus. We would then expect that the resulting phenotype may be considerably less adaptive than both phenotype A and B, as the plastic response may either be a degenerated version of phenotype B or a mixture of both phenotypes. Such nonadaptive phenotypic plasticity may be especially striking on the transcriptional level, when ancestral (adaptive responsive) and descendent lineages (less/ nonadaptive responsive) are compared under the influence of variable environmental stimuli (we summarized our predictions in Fig. 4D-F). We thus encourage studies using comparative transcriptomics in candidate radiations that determine the level of adaptive and nonadaptive plasticity from which the stage of ongoing genetic assimilation could be inferred.

Patterns of nonadaptive plasticity around colonization or invasion events have been reported frequently in previous studies, for example in a study recently published on guppies (Poecilia reticulata) (Ghalambor et al. 2015). The authors found that a pronounced nonadaptive plastic response can be induced in a putative ancestral guppy lineage (living in a high-predation environment) by an atypical stimulus (low-predation). Furthermore, the authors found that artificially formed subpopulation from this ancestral population could rapidly genetically adapt to a low-predation environment within few generations. The authors conclude that nonadaptive plasticity (rather than adaptive plasticity) may facilitate evolution. Considering that the ancestral source population lives in a high-predation environment, it may also be plausible that its high-predation phenotype is undergoing genetic assimilation. Thus, by confronting it with the low-predation stimulus, a degenerated low-predation phenotype that comes with the observed pronounced transcriptional noise may have been induced (compare to Fig. $4 \mathrm{E}$ 'Radiation 1'). Therefore, the possibility exists that the ancestral guppy population represents an already quite derived population (i.e. at an advanced stage of becoming genetically assimilated for a high-predation phenotype). Nonadaptive plasticity might thus reflect an unveiling of cryptic genetic variation by the atypical low-predation environment. Furthermore, the cryptic genetic variation has potentially originated in alternative developmental trajectories of a formerly plastic trait. Patterns of nonadaptive plastic responses in phenotypes and gene transcription can hence inform about the state of ongoing genetic assimilation. Recent investigations in cichlid fishes found evidence that predicted patterns (Fig. 4) may also be observable across African cichlid radiations (Box 4). Further examples for nonadaptive plastic responses can be found in plants: in a meta-analysis, Davidson et al. (2011) found that invasive plant species show increased levels of plasticity when compared to noninvasive species, supporting the notion that plastic species are good colonizers. However, the plastic responses of these invaders were not necessarily adaptive. The nonadaptive responses may therefore simply reflect a degenerated plastic response when an ancestrally plastic trait undergoes genetic assimilation in a new environment, as predicted by our model. 


\section{Going beyond the ancestral phenotypic range}

Thus far, this manuscript has presented and discussed studies suggesting that phenotypic plasticity can be a driver of phenotypic differentiation and lineage divergence. However, we have not yet discussed how phenotypes extend beyond ancestral plastic range (i.e. reaction norm). The modular nature of developmental trajectories (Schlosser \& Wagner 2004) is likely to facilitate diversification. It was suggested that genes contributing to plastic responses are regulated in gene regulatory modules during the plastic response, maybe by relatively few master regulators (Aubin-Horth \& Renn 2009; Schneider et al. 2014). Permanently un-induced developmental trajectories of a plastic trait that are undergoing successive degeneration can potentially be (partially) integrated into the expressed trajectory (and thus phenotype) of the trait undergoing genetic assimilation. Such cooption of regulatory circuitry may lead to phenotypes outside the range of the ancestor (Fig. 3D). Thus, characteristics specific for originally separate phenotypes may be integrated into a single derived phenotype with altered fitness. The phenotype of such a trait would no longer reflect the phenotypic range of the plastic ancestral population.

An exciting candidate for an originally plastic network undergoing genetic assimilation and subsequent cooption of degenerating developmental trajectories in a derived species can be found among the horned beetles of the Onthophagus genus. Horn size in horned beetles can be very variable, not only among species, but also between sexes and even within a sex. O. taurus exemplifies the general patterns found in the genus: males have considerably larger horns than females. However, the relative size of male horns critically depends on the food supply during an individual's larval stage: limited food supply leads to small males with disproportionally smaller horns, while food in excess induces larger males with relatively larger horns (Moczek \& Emlen 1999). The horn phenotypes are controlled in these beetles by the highly conserved doublesex ( $d s x$ ) gene pathway: alternative splice variants of $d s x$ determine both the sex of an individual and the expression of secondary sexual traits, such as the horn phenotypes (Kijimoto et al. 2009, 2012). Interestingly, a particular Onthophagus species, O. sagittarius, shows entirely altered horn development patterns compared to other species of the genus. Most notably in the context of this manuscript is that $O$. sagittarius males probably lost their ancestral plastic responsiveness towards food supply during larval stages, leaving all males with the relatively same horn size independent of body size (Kijimoto et al. 2012). Instead, they evolved two further, evolutionarily novel sets of horns: one anterior to the ancestral one that is only present in males, and one posterior to it on the thorax that is only present in females. Kijimoto et al. (2012) also found that the novel horn sets and phenotypes are due to novel functions of $d s x$ splice variants and an alteration of $d s x$ expression patterns. Horned beetles thus constitute a prime candidate to investigate 'whether' and 'how' genetic assimilation and subsequent cooption of degenerating developmental trajectories may lead to evolutionary novelty.

Diversification in plastic traits is in line with studies finding that modelled GRNs with a plastic history are more capable of adapting to new environments than those with a static history (Fierst 2011). In addition, fragments of the regulatory circuitry of degenerating developmental trajectories may start to contribute to other traits and, if a link to environmental sensitivity is maintained, facilitate the evolution of environmental sensitivity in that trait. Early in the process of such gene regulatory rewiring, involved genes and regulatory sites might experience strong selection that shapes the traits' modified phenotypes. It was previously described that relaxed selection on genes, followed by rapid evolution after being coopted in a new trait may be a mechanism of evolving phenotypic plasticity (Hunt et al. 2011; Leichty et al. 2012). This scenario thus constitutes another possible explanation for the frequent cooption of regulatory circuitry in traits that originated in completely different ones (e.g., Lee et al. 2003; Shubin et al. 2009; Peter \& Davidson 2011). In spite of the potentially positive effects of cryptic genetic variation due to noninduced degenerating developmental trajectories on the rate of evolution, such cryptic genetic variation can also have maladaptive consequences if expression is induced, e.g. by becoming a source of genetically linked diseases, as generally suggested for cryptic genetic variation (Paaby \& Rockman 2014).

In conclusion, phenotypic plasticity offers considerable advantages in species persistence in many heterogeneous environments and plastic traits can evolve, particularly via regulatory evolution. However, in unpredictable or very stable environments, genetically fixed phenotypes were shown to outperform plasticity as natural selection can act directly on the genes underlying a phenotype (Le Vinh et al. 2016). In the natural world, however, environments are rarely heterogeneous but perfectly predictable, nor perfectly stable or completely unpredictable. Therefore, selection holds most traits at a trait- and environment-specific 'equilibrium state' of genetic accommodation, which is why phenotypes of most traits can only be predicted when both genetic and environmental effects (and their interactions) are considered: $P=G+E+G x E$. Some adaptive radiations may have been the result of a flexible stem diversifying after experiencing high ecological opportunity, while others may have been driven by strong diversifying selection on a genetically fixed phenotype and diversity arose, for example, from standing genetic variation. By 
Box 4. Patterns of morphological and transcriptional plasticity in cichlids may coincide with predictions of a flexible stem in cichlids

A direct or indirect contribution of phenotypic plasticity to the adaptive radiations across cichlids has been suggested (e.g. Meyer 1987; Wimberger 1994; West-Eberhard 2003; Stauffer \& van Snick 2004 Muschick et al. 2011; Parsons et al. 2016) but few have provided specific hypotheses of the underlying processes and mechanisms (but see Wimberger 1994). Recently, it was hypothesized that similar phenotypes across radiations of the East African cichlids are not independently evolved, but rather share a common developmental trajectory that was presumably already present in their plastic common ancestor - an example of 'parallel evolution' (O'Quin et al. 2010). Insights into the molecular underpinnings of the plastic response in cichlid pharyngeal jaws as well as an increase in the knowledge of its phenotypic distributions across cichlids allow us to more specifically investigate how plasticity in key evolutionary traits has affected cichlid evolution (Muschick et al. 2012; Schneider et al. 2014). The hypothesis that a phenotypically plastic ancestral trait might have provided the baseline variability reflected today by hundreds of diverse cichlid species across several radiations (West-Eberhard 2003) challenges our understanding of how trait diversity generally evolves.

\section{Do predicted morphological and transcription patterns of plasticity fit observations in cichlids?}

An adaptive radiation initiated by a plastic ancestor would lead to a sudden increase in phenotypic diversity, followed by a gradual increase in genetic diversity, as described before. This pattern coincides with observations in modern Haplochromines: even the youngest radiation of a Great Lake (Lake Victoria) displays a plethora of phenotypes, but genetically, the species are almost indistinguishable, with fragile reproductive barriers that can easily be circumvented when conditions are manipulated (e.g. in an aquarium) (Stelkens et al. 2009). Verheyen et al. (2003) noted that the phenotypic diversity found in the Lake Victoria species flock is likely to have emerged in the very early stages of the radiation - a phenomenon that was also suggested for the Neotropical Geophagini cichlids (López-Fernández et al. 2012; Arbour \& López-Fernández 2016). Such rapid phenotypic divergence is hardly explainable assuming classical gene-mutation-based gradual phenotypic change that is assumed to require much longer time spans (Gavrilets 2003). Furthermore, our preliminary data on the level of inducible adaptive and nonadaptive phenotypic plasticity in the pharyngeal jaws of radiating East African cichlids suggest that, while two riverine species show the highest levels of adaptive plasticity, a radiating lineage from Lake Victoria and one from Lake Tanganyika show reduced and no adaptive plasticity anymore, respectively. Total morphological variation, however, is comparable between the species, suggesting that the latter shows higher degrees of nonadaptive plasticity, which is in line with the flexible stem hypothesis. Within Lake Tanganyika, the Tropheini provide further evidence for a possible flexible stem in cichlids: Kerschbaumer et al. (2011) studied four morphologically distinct populations of Tropheus moori, which have been split from each other for about 100,000 years (Sturmbauer et al. 2005). By raising broods of all four populations in a common environment, they found that plastically induced morphological variation exceeded naturally occurring population differences by a factor of 2.4. In addition, a small proportion of the variation could be linked to the genetic background. The authors also noted that plastically induced morphological patterns might have been at least partially adaptive, as experimental fish morphologies roughly coincided with the morphology of the population that lives in environments similar to the common garden. These lineages of Tropheus moorii may therefore be at an early stage of divergence where morphological niche segregation is still mostly due to adaptive plasticity and genetic assimilation has just begun to fix particular developmental trajectories.

Despite available data in line with a flexible stem in modern Haplochromines, the inferences from only morphological and genetic data are limited as long as the GRN underlying the plastic response in the cichlid pharyngeal jaw remains elusive. First efforts have been undertaken to describe this GRN in A. alluaudi (Schneider et al. 2014). Studies investigating gene expression within pharyngeal jaw bone tissue during the plastic response proposed that, throughout development, involved genes are orchestrated in gene modules in all investigated 'plastic' species, as was found for other GRNs (Aubin-Horth \& Renn 2009; Schneider et al. 2014). These gene modules are sets of functionally related genes that were observed to be jointly up- and downregulated by, potentially, only few master regulators, such as the transcription factor AP1 or the second messenger cAMP. Although data are very limited to date, the available evidence suggests that at least parts of the GRN putatively underlying ancestral pharyngeal jaw plastic development in the modern Haplochromines are preserved among plastic species. This is in 
Box 4. Continued

line with the notion that the regulatory modules may be an effective way to manage the complexity of GRNs underlying complex traits, as only relatively few master regulators are potentially necessary to control a large number of directly downstream genes (Schneider et al. 2014). Thus, during genetic assimilation, relatively few regulatory changes could have dramatic phenotypic effects, and single developmental trajectories may retain functionality while other trajectories are corrupted by mutational alterations in regulatory sites. Another very recent paper found further support for a flexible stem in Lake Malawi cichlid oral jaw morphology (Parsons et al. 2016). The authors also found that plasticity had a specific genetic signature using a QTL approach. Combined with their previously published work, the authors identified a specific gene, ptch1, to be a likely regulator of mouth plasticity.

The general importance of regulatory evolution during cichlid diversification is also reflected in their genomes, which show increased rates of regulatory evolution particularly associated with pleiotropic genes and such underlying morphological traits (Brawand et al. 2014). Our current work is focusing on understanding 'whether' and 'how' GRN degeneration may have occurred in derived cichlid lineages.

investigating the genetic, transcriptional and phenotypic patterns observable in the lineages that emerged from explosive speciation events, comparative studies can investigate the relative importance of plasticity vs. genetic determination in driving this extraordinary diversification.

\section{Summary \& concluding remarks}

In recent years, our awareness of how multifaceted and consequential the biotic and abiotic environment influences the phenotypes of all living organisms has greatly increased (Gilbert \& Epel 2015; Gilbert et al. 2015; Sul$\tan 2015)$. Phenotypic plasticity is a key response mechanism to organisms' environments. Plasticity's effects on evolutionary rates have been intensely discussed for more than a century and received renewed attention in the last decade as the molecular mechanisms become better understood and new evolutionary models have been developed (West-Eberhard 2005; Pfennig et al. 2010; Ghalambor et al. 2015; Hendry 2015; Oke et al. 2015; Levis \& Pfennig 2016).

We reviewed recent studies that suggest that phenotypic plasticity itself might be the substrate for rapid phenotypic diversification after colonization of a new environment, as (i.) it facilitates a population's persistence in a broad range of environments, (ii.) developmental trajectories can potentially be fixed, even by random mutations and (iii.) noninduced developmental trajectories that undergo degeneration contribute to population's cryptic genetic variation that can act as substrate for the evolution of further phenotypic diversity. Specifically, we propose that at least some nonadaptive plastic responses are a side product from a trait undergoing genetic assimilation. While only one developmental trajectory is fixed during genetic assimilation, alternative trajectories, which have been part of an adaptive response in an ancestor, are increasingly degenerating. Degenerating developmental trajectories might retain some of their inducibility and, if induced by atypical environmental stimulus, their plastic response becomes increasingly nonadaptive until environmental sensitivity is lost entirely. Adaptive radiations are illustrated here as emerging from a flexible stem using the model system of cichlid fishes in the boxes. Due to their diversity, common parallelism and convergence and well-described examples of phenotypic plasticity in key ecological traits, cichlids are likely to have a flexible stem in some traits that promoted their phenotypic diversity (Box 2-4).

We encourage future studies examining the occurrence and role of adaptive and nonadaptive phenotypic plasticity in traits that contributed to lineage diversification at different stages of lineage divergence and genetic assimilation. Comparative studies examining patterns of phenotypic, genetic and transcriptional variation associated with a diversifying trait across radiating lineages will further our understanding of the multifaceted contributions of phenotypic plasticity to the evolution of biological diversity.

\section{Acknowledgements}

We are grateful for funding from the Deutsche Forschungsgemeinschaft (SPP1819) and the University of Konstanz (AM, RS). AM acknowledges support from the European Research Council through ERC advanced grant GenAdap (297300). We thank Helen M. Gunter, Claudius Kratochwil, Maggie Sefton, Pooja Singh, Julian Torres-Dowdall, Adam Wilkins and Joost Woltering for valuable comments and discussions.

\section{References}

Alonso CR, Wilkins AS (2005) The molecular elements that underlie developmental evolution. Nature Reviews Genetics, 6, 709-715. 
Ancel LW, Fontana W (2000) Plasticity, evolvability, and modularity in RNA. Journal of Experimental Zoology, 288, 242283.

Arbour JH, López-Fernández H (2016) Continental cichlid radiations: functional diversity reveals the role of changing ecological opportunity in the Neotropics. Proceedings of the Royal Society B, 283, 20160556.

Aubin-Horth N, Renn SC (2009) Genomic reaction norms: using integrative biology to understand molecular mechanisms of phenotypic plasticity. Molecular Ecology, 18, 3763-3780.

Aubin-Horth N, Letcher BH, Hofmann HA (2009) Gene-expression signatures of Atlantic salmon's plastic life cycle. General and Comparative Endocrinology, 163, 278-284.

Barluenga M, Meyer A (2010) Phylogeography, colonization and population history of the Midas cichlid species complex (Amphilophus spp.) in the Nicaraguan crater lakes. BMC Evolutionary Biology, 10, 326.

Barluenga M, Stölting KN, Salzburger W, Muschick M, Meyer A (2006) Sympatric speciation in Nicaraguan crater lake cichlid fish. Nature, 439, 719-723.

Barrett RD, Schluter D (2008) Adaptation from standing genetic variation. Trends in Ecology \& Evolution, 23, 38-44.

Beaty LE, Wormington JD, Kensinger BJ et al. (2016) Shaped by the past, acting in the present: transgenerational plasticity of anti-predatory traits. Oikos, doi:10.1111/oik.03114.

Bell AM, Aubin-Horth N (2010) What can whole genome expression data tell us about the ecology and evolution of personality? Philosophical Transactions of the Royal Society B: Biological Sciences, 365, 4001-4012.

Bento G, Ogawa A, Sommer RJ (2010) Co-option of the hormone-signalling module dafachronic acid-DAF-12 in nematode evolution. Nature, 466, 494-497.

Binning S, Chapman L, Dumont J (2010) Feeding and breathing: trait correlations in an African cichlid fish. Journal of Zoology, 282, 140-149.

Bradshaw AD (1965) Evolutionary significance of phenotypic plasticity in plants. Advances in Genetics, 13, 115-155.

Brawand D, Wagner CE, Li YI et al. (2014) The genomic substrate for adaptive radiation in African cichlid fish. Nature, 513, 375-381.

Brewer MS, Carter RA, Croucher PJ, Gillespie RG (2015) Shifting habitats, morphology, and selective pressures: developmental polyphenism in an adaptive radiation of Hawaiian spiders. Evolution, 69, 162-178.

Britten RJ, Davidson EH (1971) Repetitive and non-repetitive DNA sequences and a speculation on the origins of evolutionary novelty. Quarterly Review of Biology, 46, 111-138.

Cahan SH, Julian GE, Rissing SW et al. (2004) Loss of phenotypic plasticity generates genotype-caste association in harvester ants. Current Biology, 14, 2277-2282.

Carroll SB (2008) Evo-devo and an expanding evolutionary synthesis: a genetic theory of morphological evolution. Cell, 134, 25-36.

Chapman L, Galis F, Shinn J (2001) Phenotypic plasticity and the possible role of genetic assimilation: Hypoxia-induced trade-offs in the morphological traits of an African cichlid. Ecology Letters, 3, 387-393.

Chevin L-M, Lande R, Mace GM (2010) Adaptation, plasticity, and extinction in a changing environment: towards a predictive theory. PLoS Biology, 8, e1000357.
Colombo M, Diepeveen ET, Muschick M et al. (2013) The ecological and genetic basis of convergent thick-lipped phenotypes in cichlid fishes. Molecular Ecology, 22, 670-684.

Cotman CW, Berchtold NC (2002) Exercise: a behavioral intervention to enhance brain health and plasticity. Trends in Neurosciences, 25, 295-301.

Davidson EH, Erwin DH (2006) Gene regulatory networks and the evolution of animal body plans. Science, 311, 796-800.

Davidson AM, Jennions M, Nicotra AB (2011) Do invasive species show higher phenotypic plasticity than native species and if so, is it adaptive? A meta-analysis. Ecology Letters, 14, 419-431.

DeWitt TJ, Scheiner SM (2004) Phenotypic Plasticity: Functional and Conceptual Approaches. Oxford University Press, Oxford.

DeWitt TJ, Sih A, Wilson DS (1998) Costs and limits of phenotypic plasticity. Trends in Ecology \& Evolution, 13, 77-81.

Dieckmann U, Doebeli M (1999) On the origin of species by sympatric speciation. Nature, 400, 354-357.

Dodson S (1988) The ecological role of chemical stimuli for the zooplankton: Predator-avoidance behavior in Daphnia. Limnology and Oceanography, 33, 1431-1439.

Doebeli M, Dieckmann U (2003) Speciation along environmental gradients. Nature, 421, 259-264.

Draghi JA, Whitlock MC (2012) Phenotypic plasticity facilitates mutational variance, genetic variance, and evolvability along the major axis of environmental variation. Evolution, 66, 2891-2902.

Ehrenreich IM, Pfennig DW (2015) Genetic assimilation: a review of its potential proximate causes and evolutionary consequences. Annals of Botany, 115, 769-779.

Elmer KR, Reggio C, Wirth T et al. (2009) Pleistocene desiccation in East Africa bottlenecked but did not extirpate the adaptive radiation of Lake Victoria haplochromine cichlid fishes. Proceedings of the National Academy of Sciences USA, 106, 13404-13409.

Elmer KR, Kusche H, Lehtonen TK, Meyer A (2010) Local variation and parallel evolution: morphological and genetic diversity across a species complex of neotropical crater lake cichlid fishes. Philosophical Transactions of the Royal Society B: Biological Sciences, 365, 1763-1782.

Emlen DJ (1994) Environmental control of horn length dimorphism in the beetle Onthophagus acuminatus (Coleoptera: Scarabaeidae). Proceedings of the Royal Society of London. Series B: Biological Sciences, 256, 131-136.

Fierst J (2011) A history of phenotypic plasticity accelerates adaptation to a new environment. Journal of Evolutionary Biology, 24, 1992-2001.

Fisher RA (1930) The Genetical Theory of Natural Selection: A Complete Variorum Edition. Oxford University Press, Oxford.

Foster S, Wund M, Graham M et al. (2015) Iterative development and the scope for plasticity: contrasts among trait categories in an adaptive radiation. Heredity, 115, 335-348.

Gavrilets S (2003) Perspective: models of speciation: what have we learned in 40 years? Evolution, 57, 2197-2215.

Ghalambor CK, McKay JK, Carroll SP, Reznick DN (2007) Adaptive versus non-adaptive phenotypic plasticity and the potential for contemporary adaptation in new environments. Functional Ecology, 21, 394- 407.

Ghalambor CK, Hoke KL, Ruell EW et al. (2015) Non-adaptive plasticity potentiates rapid adaptive evolution of gene expression in nature. Nature, 525, 372-375. 
Gibson G (2008) The environmental contribution to gene expression profiles. Nature Reviews Genetics, 9, 575-581.

Gilbert SF, Epel D (2009) Ecological Developmental Biology. Sinauer Associates, Sunderland.

Gilbert SF, Epel D (2015) Ecological Developmental Biology: The Environmental Regulation of Development, Health, and Evolution. Sinauer Associates, Incorporated, Sunderland.

Gilbert SF, Bosch TC, Ledón-Rettig C (2015) Eco-Evo-Devo: developmental symbiosis and developmental plasticity as evolutionary agents. Nature Reviews Genetics, 16, 611-622.

Gittenberger E (1991) What about non-adaptive radiation? Biological Journal of the Linnean Society, 43, 263-272.

Greenwood P (1964) Environmental effects on the pharyngeal mill of a cichlid fish, Astatoreochromis alluaudi, and their taxonomic implications. Proceedings of the Linnean Society of London, 176, 1-10.

Gunter HM, Fan S, Xiong F et al. (2013) Shaping development through mechanical strain: the transcriptional basis of dietinduced phenotypic plasticity in a cichlid fish. Molecular Ecology, 22, 4516-4531.

Gutteling E, Riksen J, Bakker J, Kammenga J (2007) Mapping phenotypic plasticity and genotype-environment interactions affecting life-history traits in Caenorhabditis elegans. Heredity, 98, 28-37.

Hayden EJ, Ferrada E, Wagner A (2011) Cryptic genetic variation promotes rapid evolutionary adaptation in an RNA enzyme. Nature, 474, 92-95.

Hendry AP (2015) Key questions on the role of phenotypic plasticity in eco-evolutionary dynamics. Journal of Heredity, 107, 25-41.

Hinman VF, Yankura KA, McCauley BS (2009) Evolution of gene regulatory network architectures: examples of subcircuit conservation and plasticity between classes of echinoderms. Biochimica et Biophysica Acta (BBA)-Gene Regulatory Mechanisms, 1789, 326-332.

Hoekstra HE, Coyne JA (2007) The locus of evolution: evo devo and the genetics of adaptation. Evolution, 61, 995-1016.

Hofmann HA (2003) Functional genomics of neural and behavioral plasticity. Journal of Neurobiology, 54, 272-282.

Hölldobler B (1990) The Ants. Harvard University Press, Cambridge.

Hoogerhoud R (1984) A taxonomic reconsideration of the haplochromine genera Gaurochromis Greenwood, 1980 and Labrochromis Regan, 1920 (Pisces, Cichlidae). Netherlands Journal of Zoology, 34, 539-565.

Hoogerhoud R (1986) The adverse effects of shell ingestion for Molluscivorous cichlids, a constructional morphological approach. Netherlands Journal of Zoology, 37, 3-4.

Hunt BG, Ometto L, Wurm Y et al. (2011) Relaxed selection is a precursor to the evolution of phenotypic plasticity. Proceedings of the National Academy of Sciences USA, 108, 15936-15941.

Iwasaki WM, Tsuda ME, Kawata M (2013) Genetic and environmental factors affecting cryptic variations in gene regulatory networks. BMC Evolutionary Biology, 13, 1.

Jablonka E, Lamb MJ (2007) The expanded evolutionary synthesis-a response to Godfrey-Smith, Haig, and West-Eberhard. Biology E Philosophy, 22, 453-472.

Jarvela AMC, Hinman VF (2015) Evolution of transcription factor function as a mechanism for changing metazoan developmental gene regulatory networks. EvoDevo, 6, 3 .
Kawecki TJ (1997) Sympatric speciation via habitat specialization driven by deleterious mutations. Evolution, 51, 1751-1763.

Kerschbaumer M, Postl L, Koch M, Wiedl T, Sturmbauer C (2011) Morphological distinctness despite large-scale phenotypic plasticity-analysis of wild and pond-bred juveniles of allopatric populations of Tropheus moorii. Naturwissenschaften, 98, 125-134.

Kijimoto T, Costello J, Tang Z, Moczek AP, Andrews J (2009) EST and microarray analysis of horn development in Onthophagus beetles. BMC Genomics, 10, 1.

Kijimoto T, Moczek AP, Andrews J (2012) Diversification of doublesex function underlies morph-, sex-, and species-specific development of beetle horns. Proceedings of the National Academy of Sciences USA, 109, 20526-20531.

King M-C, Wilson AC (1975) Evolution at two Levels in Humans and Chimpanzees. Science, 188, 107-116.

Kocher TD (2004) Adaptive evolution and explosive speciation: the cichlid fish model. Nature Reviews Genetics, 5, 288-298.

Kocher TD, Conroy JA, McKaye KR, Stauffer JR (1993) Similar morphologies of cichlid fish in Lakes Tanganyika and Malawi are due to convergence. Molecular Phylogenetics and Evolution, 2, 158-165.

Kondrashov AS, Kondrashov FA (1999) Interactions among quantitative traits in the course of sympatric speciation. Nature, 400, 351-354.

Lahti DC, Johnson NA, Ajie BC et al. (2009) Relaxed selection in the wild. Trends in Ecology \& Evolution, 24, 487-496.

Le Vinh TJ, Vandenbrooks J, Angilletta Jr M (2016) Developmental plasticity evolved according to specialist-generalist trade-offs in experimental populations of Drosophila melanogaster. Biology Letters, 12, doi: 10.1098/rsbl.2016.0379.

Lee PN, Callaerts P, de Couet HG, Martindale MQ (2003) Cephalopod Hox genes and the origin of morphological novelties. Nature, 424, 1061-1065.

Leichty AR, Pfennig DW, Jones CD, Pfennig KS (2012) Relaxed genetic constraint is ancestral to the evolution of phenotypic plasticity. Integrative and Comparative Biology, 52, 16-30.

Levis NA, Pfennig DW (2016) Evaluating 'plasticity-first'evolution in nature: key criteria and empirical approaches. Trends in Ecology \& Evolution, 31, 563-574.

Lopez-Fernández H, Honeycutt RL, Winemiller KO (2005) Molecular phylogeny and evidence for an adaptive radiation of geophagine cichlids from South America (Perciformes: Labroidei). Molecular Phylogenetics and Evolution, 34, 227-244.

López-Fernández $\mathrm{H}$, Winemiller $\mathrm{KO}$, Montaña $\mathrm{C}$, Honeycutt RL (2012) Diet-morphology correlations in the radiation of South American geophagine cichlids (Perciformes: Cichlidae: Cichlinae). PLoS ONE, 7, e33997.

Losos JB, Creer DA, Glossip D et al. (2000) Evolutionary implications of phenotypic plasticity in the hindlimb of the lizard Anolis sagrei. Evolution, 54, 301-305.

Lundsgaard-Hansen B, Matthews B, Vonlanthen P, Taverna A, Seehausen O (2013) Adaptive plasticity and genetic divergence in feeding efficiency during parallel adaptive radiation of whitefish (Coregonus spp.). Journal of Evolutionary Biology, 26, 483-498.

Manousaki T, Hull PM, Kusche H et al. (2013) Parsing parallel evolution: ecological divergence and differential gene expression in the adaptive radiations of thick-lipped Midas cichlid fishes from Nicaragua. Molecular Ecology, 22, 650-669. 
Masel J, King OD, Maughan H (2007) The loss of adaptive plasticity during long periods of environmental stasis. The American Naturalist, 169, 38.

Mayo AE, Setty Y, Shavit S, Zaslaver A, Alon U (2006) Plasticity of the cis-regulatory input function of a gene. PLoS Biology, 4, 555 .

Mazzarella AB, Voje KL, Hansson TH, Taugbøl A, Fischer B (2015) Strong and parallel salinity-induced phenotypic plasticity in one generation of threespine stickleback. Journal of Evolutionary Biology, 28, 667-677.

Meyer A (1987) Phenotypic plasticity and heterochrony in Cichlasoma managuense (Pisces, Chichlidae) and their implications for speciation in Cichlid fishes. Evolution, 41, 13571369.

Meyer A (1989) Cost of morphological specialization: feeding performance of the two morphs in the trophically polymorphic cichlid fish, Cichlasoma citrinellum. Oecologia, 80, 431-436.

Meyer A (1993a) Phylogenetic relationships and evolutionary processes in East African cichlid fishes. Trends in Ecology \& Evolution, 8, 279-284.

Meyer A (1993b) Trophic polymorphisms in cichlid fish: do they represent intermediate steps during sympatric speciation and explain their rapid adaptive radiation. Trends in Ichthyology, 7, 257-266.

Meyer A, Kocher TD, Basasibwaki P, Wilson AC (1990) Monophyletic origin of Lake Victoria cichlid fishes suggested by mitochondrial DNA sequences. Nature, 347, 550-553.

Mirouze M, Paszkowski J (2011) Epigenetic contribution to stress adaptation in plants. Current Opinion in Plant Biology, 14, 267-274.

Moczek A, Emlen D (1999) Proximate determination of male horn dimorphism in the beetle Ontophagus taurus (Coleoptera: Scarabaeidae). Journal of Evolutionary Biology, 12, 27-37.

Moczek AP, Kijimoto T (2014) Development and evolution of insect polyphenisms: novel insights through the study of sex determination mechanisms. Current Opinion in Insect Science, 1, 52-58.

Murren CJ, Auld JR, Callahan H et al. (2015) Constraints on the evolution of phenotypic plasticity: limits and costs of phenotype and plasticity. Heredity, 115, 293-301.

Muschick M, Barluenga M, Salzburger W, Meyer A (2011) Adaptive phenotypic plasticity in the Midas cichlid fish pharyngeal jaw and its relevance in adaptive radiation. $B M C$ Evolutionary Biology, 11, 116.

Muschick M, Indermaur A, Salzburger W (2012) Convergent evolution within an adaptive radiation of cichlid fishes. Current Biology, 22, 2362-2368.

Nei M, Maruyama T, Wu C-I (1983) Models of evolution of reproductive isolation. Genetics, 103, 557-579.

Newman RA (1992) Adaptive plasticity in amphibian metamorphosis. BioScience, 42, 671-678.

Nicotra AB, Atkin OK, Bonser SP et al. (2010) Plant phenotypic plasticity in a changing climate. Trends in Plant Science, 15, 684-692.

Nijhout HF (2003) Development and evolution of adaptive polyphenisms. Evolution \& Development, 5, 9-18.

Nijhout HF (2015) A Developmental-Physiological Perspective on the Development and Evolution of Phenotypic Plasticity. In: Conceptual Change in Biology (eds Love AC), pp. 147-173. Springer, Dordrecht, Netherlands.
Nussey DH, Postma E, Gienapp P, Visser ME (2005) Selection on heritable phenotypic plasticity in a wild bird population. Science, 310, 304-306.

Nylin S, Gotthard K (1998) Plasticity in life-history traits. Annual Review of Entomology, 43, 63-83.

Nylin S, Wahlberg N (2008) Does plasticity drive speciation? Host-plant shifts and diversification in nymphaline butterflies (Lepidoptera: Nymphalidae) during the tertiary. Biological Journal of the Linnean Society, 94, 115-130.

Odling-Smee FJ, Laland KN, Feldman MW (1996) Niche construction. The American Naturalist, 147, 641-648.

Oke KB, Bukhari M, Kaeuffer R et al. (2015) Does plasticity enhance or dampen phenotypic parallelism? A test with three lake-stream stickleback pairs. Journal of Evolutionary Biology, 29, 126-143.

O'Quin KE, Hofmann CM, Hofmann HA, Carleton KL (2010) Parallel evolution of opsin gene expression in African cichlid fishes. Molecular Biology and Evolution, 27, 2839-2854.

Orizaola G, Laurila A (2016) Developmental plasticity increases at the northern range margin in a warm-dependent amphibian. Evolutionary Applications, 9, 471-478.

Ostwald W (1904) Experimentelle Untersuchungen über den Saisonpolymorphismus bei Daphniden. Development Genes and Evolution, 18, 415-451.

Paaby AB, Rockman MV (2014) Cryptic genetic variation: evolution's hidden substrate. Nature Reviews Genetics, 15, 247258.

Parsons KJ, Concannon M, Navon D et al. (2016) Foraging environment determines the genetic architecture and evolutionary potential of trophic morphology in cichlid fishes. Molecular Ecology, doi: 10.1111/mec.13801.

Peter IS, Davidson EH (2011) Evolution of gene regulatory networks controlling body plan development. Cell, 144, 970-985.

Pfennig DW, Ehrenreich IM (2014) Towards a gene regulatory network perspective on phenotypic plasticity, genetic accommodation and genetic assimilation. Molecular Ecology, 23, 4438-4440.

Pfennig DW, McGee M (2010) Resource polyphenism increases species richness: a test of the hypothesis. Philosophical Transactions of the Royal Society B: Biological Sciences, 365, 577-591.

Pfennig DW, Pfennig KS (2012) Evolution's Wedge: Competition and the Origins of Diversity. University of California Press, Berkeley, UK.

Pfennig DW, Reeve HK, Sherman PW (1993) Kin recognition and cannibalism in spadefoot toad tadpoles. Animal Behaviour, 46, 87-94.

Pfennig DW, Murphy PJ, Wiens J (2002) How fluctuating competition and phenotypic plasticity mediate species divergence. Evolution, 56, 1217-1228.

Pfennig DW, Wund MA, Snell-Rood EC et al. (2010) Phenotypic plasticity's impacts on diversification and speciation. Trends in Ecology \& Evolution, 25, 459-467.

Piálek L, Říčan O, Casciotta J, Almirón A, Zrzavý J (2012) Multilocus phylogeny of Crenicichla (Teleostei: Cichlidae), with biogeography of the C. lacustris group: species flocks as a model for sympatric speciation in rivers. Molecular Phylogenetics and Evolution, 62, 46-61.

Pigliucci M, Müller GB (2010) Elements of an extended evolutionary synthesis. Evolution: The Extended Synthesis, pp. 3-17. MIT Press, Cambridge. 
Pigliucci M, Murren CJ (2003) Perspective: Genetic assimilation and a possible evolutionary paradox: can macroevolution sometimes be so fast as to pass us by? Evolution, 57, 1455-1464.

Pigliucci M, Murren CJ, Schlichting CD (2006) Phenotypic plasticity and evolution by genetic assimilation. Journal of Experimental Biology, 209, 2362-2367.

Price TD, Qvarnström A, Irwin DE (2003) The role of phenotypic plasticity in driving genetic evolution. Proceedings of the Royal Society of London. Series B: Biological Sciences, 270, 14331440.

Prud'homme B, Gompel N, Carroll SB (2007) Emerging principles of regulatory evolution. Proceedings of the National Academy of Sciences USA, 104, 8605-8612.

Ragsdale EJ, Müller MR, Rödelsperger C, Sommer RJ (2013) A developmental switch coupled to the evolution of plasticity acts through a sulfatase. Cell, 155, 922-933.

Renn SCP, Schumer ME (2013) Genetic accommodation and behavioural evolution: insights from genomic studies. Animal Behaviour, 85, 1012-1022.

Richards CL, Bossdorf O, Muth NZ, Gurevitch J, Pigliucci M (2006) Jack of all trades, master of some? On the role of phenotypic plasticity in plant invasions. Ecology Letters, 9, 981993.

Robinson BW, Parsons KJ (2002) Changing times, spaces, and faces: tests and implications of adaptive morphological plasticity in the fishes of northern postglacial lakes. Canadian Journal of Fisheries and Aquatic Sciences, 59, 1819-1833.

Rüber L, Adams D (2001) Evolutionary convergence of body shape and trophic morphology in cichlids from Lake Tanganyika. Journal of Evolutionary Biology, 14, 325-332.

Rundell RJ, Price TD (2009) Adaptive radiation, nonadaptive radiation, ecological speciation and nonecological speciation. Trends in Ecology \& Evolution, 24, 394-399.

Rutherford SL, Lindquist S (1998) Hsp90 as a capacitor for morphological evolution. Nature, 396, 336-342.

Salzburger W, Mack T, Verheyen E, Meyer A (2005) Out of Tanganyika: genesis, explosive speciation, key-innovations and phylogeography of the haplochromine cichlid fishes. BMC Evolutionary Biology, 5, 17.

Scheiner SM (1993) Genetics and evolution of phenotypic plasticity. Annual Review of Ecology and Systematics, 24, 35-68.

Scheiner SM, Holt RD (2012) The genetics of phenotypic plasticity. X. Variation versus uncertainty. Ecology and Evolution, 2, 751-767.

Schlichting CD (1986) The evolution of phenotypic plasticity in plants. Annual Review of Ecology and Systematics, 17, 667-693.

Schlichting C (2004) The role of phenotypic plasticity in diversification. In: Phenotypic Plasticity: Functional and Conceptual Approaches, DeWitt TJ, Scheiner SM, pp. 191-200. Oxford University Press, Oxford.

Schlichting CD, Pigliucci M (1993) Control of phenotypic plasticity via regulatory genes. American Naturalist, 142, 366-370.

Schlichting CD, Pigliucci M (1998) Phenotypic Evolution: A Reaction Norm Perspective. Sinauer Associates Incorporated, Sunderland.

Schlichting CD, Wund MA (2014) Phenotypic plasticity and epigenetic marking: an assessment of evidence for genetic accommodation. Evolution, 68, 656-672.

Schlosser G, Wagner GP (2004) Modularity in Development and Evolution. University of Chicago Press, Chicago.
Schluter D (1993) Adaptive radiation in sticklebacks: size, shape, and habitat use efficiency. Ecology, 74, 699-709.

Schluter D (2000) The Ecology of Adaptive Radiation. OUP, Oxford.

Schneider RF, Li Y, Meyer A, Gunter HM (2014) Regulatory gene networks that shape the development of adaptive phenotypic plasticity in a cichlid fish. Molecular Ecology, 23, $4511-4526$.

Schön I, Martens K (2004) Adaptive, pre-adaptive and nonadaptive components of radiations in ancient lakes: a review. Organisms Diversity \& Evolution, 4, 137-156.

Schwander T, Leimar O (2011) Genes as leaders and followers in evolution. Trends in Ecology \& Evolution, 26, 143-151.

Seehausen O (2006) African cichlid fish: a model system in adaptive radiation research. Proceedings of the Royal Society of London B: Biological Sciences, 273, 1987-1998.

Serobyan V, Ragsdale EJ, Müller MR, Sommer RJ (2013) Feeding plasticity in the nematode Pristionchus pacificus is influenced by sex and social context and is linked to developmental speed. Evolution \& Development, 15, 161-170.

Serobyan V, Xiao H, Namdeo S et al. (2016) Chromatin remodelling and antisense-mediated up-regulation of the developmental switch gene eud-1 control predatory feeding plasticity. Nature Communications, 7, doi:10.1038/ncomms12337.

Shiga M, Takigawa I, Mamitsuka H (2007) Annotating gene function by combining expression data with a modular gene network. Bioinformatics, 23, i468-i478.

Shubin N, Tabin C, Carroll S (2009) Deep homology and the origins of evolutionary novelty. Nature, 457, 818-823.

Sikkink KL, Reynolds RM, Ituarte CM, Cresko WA, Phillips PC (2014) Rapid evolution of phenotypic plasticity and shifting thresholds of genetic assimilation in the nematode Caenorhabditis remanei. G3: Genes| Genomes| Genetics, 4, 1103-1112.

Simpson SJ, McCaffery A, Haegele BF (1999) A behavioural analysis of phase change in the desert locust. Biological Reviews, 74, 461-480.

Smith JM (1989) Evolutionary Genetics. Oxford University Press, Oxford.

Smits J, Witte F, Veen F (1996) Functional changes in the anatomy of the pharyngeal jaw apparatus of Astatoreochromis alluaudi (Pisces, Cichlidae), and their effects on adjacent structures. Biological Journal of the Linnean Society, 59, 389-409.

Snell-Rood EC, Van Dyken JD, Cruickshank T, Wade MJ, Moczek AP (2010) Toward a population genetic framework of developmental evolution: the costs, limits, and consequences of phenotypic plasticity. BioEssays, 32, 71-81.

Spight TM, Emlen J (1976) Clutch sizes of two marine snails with a changing food supply. Ecology, 57, 1162-1178.

Stauffer JR, van Snick GE (2004) Phenotypic plasticity: its role in trophic radiation and explosive speciation in cichlids (Teleostei: Cichlidae). Animal Biology, 54, 137-158.

Stebbins Jr C (ed) (1950) Variation and evolution in plants. In: Variation and Evolution in Plants, Columbia Univ. Press, New York.

Stelkens RB, Schmid C, Selz O, Seehausen O (2009) Phenotypic novelty in experimental hybrids is predicted by the genetic distance between species of cichlid fish. BMC Evolutionary Biology, 9, 283.

Stiassny ML, Meyer A (1999) Cichlids of the rift lakes. Scientific American - American Edition, 280, 64-69. 
Sturmbauer C, Koblmüller S, Sefc KM, Duftner N (2005) Phylogeographic history of the genus Tropheus, a lineage of rockdwelling cichlid fishes endemic to Lake Tanganyika. Hydrobiologia, 542, 335-366.

Sultan S (2015) Organism \& Environment, 1st edn. Oxford University Press, New York, New York.

Susoy V, Ragsdale EJ, Kanzaki N, Sommer RJ (2015) Rapid diversification associated with a macroevolutionary pulse of developmental plasticity. Elife, 4, e05463.

Swanson BO, Gibb AC, Marks JC, Hendrickson DA (2003) Trophic polymorphism and behavioral differences decrease intraspecific competition in a cichlid, Herichthys minckleyi. Ecology, 84, 1441-1446.

Tebbich S, Sterelny K, Teschke I (2010) The tale of the finch: adaptive radiation and behavioural flexibility. Philosophical Transactions of the Royal Society of London B: Biological Sciences, 365, 1099-1109.

Thibert-Plante X, Hendry A (2011) The consequences of phenotypic plasticity for ecological speciation. Journal of Evolutionary Biology, 24, 326-342.

Torres-Dowdall J, Handelsman CA, Reznick DN, Ghalambor CK (2012) Local adaptation and the evolution of phenotypic plasticity in Trinidadian guppies (Poecilia reticulata). Evolution, 66, 3432-3443.

Trewavas E, Green J, Corbet SA (1972) Ecological studies on crater lakes in West Cameroon fishes of Barombi Mbo. Journal of Zoology, 167, 41-95.

Turcotte MM, Levine JM (2016) Phenotypic Plasticity and Species Coexistence. Trends in Ecology \& Evolution, 31, 803-813.

Turner GF, Seehausen O, Knight ME, Allender CJ, Robinson RL (2001) How many species of cichlid fishes are there in African lakes? Molecular Ecology, 10, 793-806.

Van Laere A-S, Nguyen M, Braunschweig M et al. (2003) A regulatory mutation in IGF2 causes a major QTL effect on muscle growth in the pig. Nature, 425, 832-836.

Verheyen E, Salzburger W, Snoeks J, Meyer A (2003) Origin of the superflock of cichlid fishes from Lake Victoria, East Africa. Science, 300, 325-329.

Visser ME, Holleman LJ, Caro SP (2009) Temperature has a causal effect on avian timing of reproduction. Proceedings of the Royal Society B: Biological Sciences, 276, 2323-2331.

Waddington $\mathrm{CH}$ (1942) Canalization of development and the inheritance of acquired characters. Nature, 150, 563-565.

Waddington CH (1953) Genetic assimilation of an acquired character. Evolution, 7, 118-126.

Waddington CH (1961) Genetic assimilation. Advances in Genetics, 10, 257-293.

Wagner A (2008) Robustness and evolvability: a paradox resolved. Proceedings of the Royal Society of London B: Biological Sciences, 275, 91-100.
Wagner GP, Pavlicev M, Cheverud JM (2007) The road to modularity. Nature Reviews Genetics, 8, 921-931.

Warner RR, Swearer SE (1991) Social control of sex change in the bluehead wrasse, Thalassoma bifasciatum (Pisces: Labridae). The Biological Bulletin, 181, 199-204.

Wellborn GA, Langerhans RB (2015) Ecological opportunity and the adaptive diversification of lineages. Ecology and Evolution, 5, 176-195.

West-Eberhard MJ (1989) Phenotypic plasticity and the origins of diversity. Annual Review of Ecology and Systematics, 20, 249-278.

West-Eberhard MJ (2003) Developmental Plasticity and Evolution. Oxford University Press, Oxford.

West-Eberhard MJ (2005) Developmental plasticity and the origin of species differences. Proceedings of the National Academy of Sciences of the United States of America, 102, 65436549.

Whitelaw NC, Whitelaw E (2006) How lifetimes shape epigenotype within and across generations. Human Molecular Genetics, 15, R131-R137.

Williams GC (1966) Adaptation and Natural Selection: A Critique of Some Current Evolutionary Thought. Princeton University Press, Princton, New Jersey.

Wimberger PH (1994) Trophic polymorphisms, plasticity, and speciation in vertebrates. Advances in Fish Foraging Theory and Ecology, 18, 19-43.

Woltereck R (1913) Beitrag zur Analyse der „Vererbung erworbener Eigenschaften": Transmutation und Präinduktion beiDaphnia. Molecular and General Genetics MGG, 9, 146-155.

Wray GA (2007) The evolutionary significance of cis-regulatory mutations. Nature Reviews Genetics, 8, 206-216.

Wund MA, Baker JA, Clancy B, Golub JL, Foster SA (2008) A test of the flexible stem model of evolution: ancestral plasticity, genetic accommodation, and morphological divergence in the threespine stickleback radiation. The American Naturalist, 172, 449-462.

Yeh PJ, Price TD (2004) Adaptive phenotypic plasticity and the successful colonization of a novel environment. The American Naturalist, 164, 531-542.

Young KA, Snoeks J, Seehausen O (2009) Morphological diversity and the roles of contingency, chance and determinism in African cichlid radiations. PLoS ONE, 4, e4740.

Zhang XS (2005) Evolution and maintenance of the environmental component of the phenotypic variance: benefit of plastic traits under changing environments. The American Naturalist, 166, 569-580.

Zimova M, Mills LS, Nowak JJ (2016) High fitness costs of climate change-induced camouflage mismatch. Ecology Letters, 19, 299-307. 